\section{FACTORES DETERMINANTES DE LA ADHERENCIA DEPORTIVA JUVENIL: APORTACIONES DESDE LOS ITINERARIOS DE OCIO DEPORTIVO}

\section{Fatores Determinantes da Aderência Esportiva Juvenil: Contributos dos Itinerários dos Esportes de Lazer}

\author{
Determinants of Adherence in Youth Sports: Contributions from \\ the Itineraries of leisure sports
}

\section{Déterminants de la Jeunesse Sportive d'Adhérence: Les Con- tributions des Itinéraires de Sports de Loisirs}

\begin{abstract}
Resumen
Inspirada en la teoría del ocio humanista este artículo presenta los resultados de una investigación que, a través del estudio de los itinerarios de ocio deportivo juveniles, busca esclarecer los factores implicados en la formación de estilos de ocio deportivo duraderos, contribuyendo así a la conversión de la acción deportiva en un factor de desarrollo personal y calidad de vida. Conscientes de la relevancia de este hecho y de sus implicaciones, la reconstrucción de las experiencias de ocio deportivo de una muestra de 383 jóvenes de Bizkaia entre 1995 y 2005, la identificación de sus itinerarios y los factores psicosociales que propician su participación deportiva y su continuidad, constituyen el corpus empírico del estudio realizado. Los resultados confirman que existen en relación a la práctica deportiva distintos patrones evolutivos que, modulados por factores psicosociales cuyo impacto en las decisiones sobre continuidad o abandono deportivo ha sido probado, permiten vislumbrar la futura dirección de los itinerarios. El conocimiento de tales factores permite contemplar la intervención de los agentes implicados como una posibilidad real dirigida a instaurar la práctica deportiva y su continuidad en los estilos de vida contemporáneos.
\end{abstract}

Palabras clave: ocio; jóvenes; itinerarios de ocio; promoción; práctica deportiva.

\section{Resumo}

Inspirado na teoria humanística do lazer, este artigo, apresenta os resultados de uma investigação que, mediante o estudo de itinerários de lazer esportivo juvenil, procura esclarecer os fatores envolvidos na formação de estilos de lazer esportivo duradouros, contribuindo assim para a conversão da ação esportiva, num fator de desenvolvimento pessoal e de qualidade de vida. Conscientes da importância deste fato e das suas implicações, a reconstrução de experiências esportivas de lazer, recorreu-se a uma amostra de 383 jovens de Biscaia, entre 1995 e 2005 , através da identificação dos seus itinerários e, os fatores psicossociais que propiciam a sua participação esportiva e a sua continuidade, constituem o corpus empírico da investigação realizada. Os resultados confirmam que em relação à prática esportiva, existem diferentes padrões evolutivos, modulados por fatores psicossociais, cujo impacto nas decisões sobre a continuidade ou abandono esportivo foi provado, permitindo vislumbrar a futura direção dos itinerários. O conhecimento de tais fatores, permite-nos descortinar a intervenção dos agentes implicados como uma real possibilidade de estabelecer a prática esportiva, e sua continuidade nos estilos de vida contemporâneos.

Palavras-chave: lazer; juventude; rotas de lazer; promoção; prática de esportes.

\section{Abstract}

TInspired by the humanistic theory of leisure, this paper presents the results of an investigation
Artigo Original
1) Doctora en Ocio y Potencial Humano por la Universidad de Deusto. Su labor investigadora se ha centrado en el estudio del ocio y de la práctica deportiva desde la Psicología, especializándose en el tema de los beneficios, barreras e itinerarios de ocio a lo largo de la vida.
Recebido em: 09/07/2012 Revisado em: 25/06/2014 Aceito em: 09/07/2014 
through the study of youth sports leisure itineraries, seeking to clarify the factors involved in the formation of durable sports leisure styles, thus contributing to the conversion of sports action by a factor of personal development and quality of life. Aware of the importance of this fact and its implications, the reconstruction of sports entertainment experiences of a sample of 383 youths of Bizkaia (Spain) between 1995 and 2005, the identification of their schedules and psychosocial factors that encourage sports participation and continuity constitute the empirical corpus study. The results confirm that exist in relation to sport different evolutionary patterns, modulated by psychosocial factors which impact on decisions about continuing or abandoning sports has been proven, a glimpse into the future direction of the routes. Knowledge of these factors allows us to envision the involvement of stakeholders as a real possibility led to the establishment of the sport and its continuity in contemporary lifestyles.

Keywords: leisure; youth; leisure itineraries; promotion; sports practice.

\section{Résumé}

Inspiré par la théorie humaniste de loisirs, cet article présente les résultats d'une enquête à travers l'étude de sports de loisirs de la jeunesse routes, cherchant à clarifier les facteurs impliqués dans la formation des sportifs durables styles de loisirs, contribuant ainsi à la conversion de les sports d'action d'un facteur de développement personnel et la qualité de vie. Conscient de l'importance de ce fait et ses conséquences, la reconstruction des expériences de divertissement de sport d'un échantillon de 383 jeunes de Gascogne entre 1995 et 2005, l'identification de leurs horaires et les facteurs psychosociaux qui favorisent la participation aux sports et à la continuité constituent l'étude de corpus empiriques. Les résultats confirment que existent dans le domaine du sport différents modèles évolutifs, modulées par des facteurs psychosociaux qui influent sur les décisions concernant la poursuite ou l'abandon de sport a fait ses preuves, un aperçu de l'orientation future des routes. La connaissance de ces facteurs nous permet d'envisager l'implication des parties prenantes comme une possibilité réelle conduit à la création de ce sport et sa continuité dans les modes de vie contemporains.

Mots-clés: loisirs; de la jeunesse; des itinéraires de loisirs; de la promotion; la pratique des sports.

Identificar las claves para consolidar el hábito deportivo en los estilos de vida de la ciudadanía ha sido y es una preocupación para las instituciones desde hace décadas. Esta preocupación es aún mayor actualmente debido al progresivo debilitamiento de los estilos de ocio físicamente activos en el colectivo joven. Estudios recientes confirman la pérdida de protagonismo de las prácticas deportivas en los estilos de ocio juveniles (Cecchini, Méndez, \& Contreras, 2005; Deloitte, 2009; Fernández, 2007; García Ferrando, 2006, 2011). El predominio de conductas de ocio sedentarias entre los jóvenes así como el abandono definitivo de sus prácticas deportivas son dos de los principales problemas a los que deben enfrentarse las instituciones y profesionales que quieran promover la adherencia deportiva en este colectivo (García Ferrando, 2011; Monteagudo, 2011).

La mayoría de los estudios que profundizan en el descenso de la práctica deportiva juvenil analizan sus consecuencias en términos de salud (Bailey et al., 2005; Balaguer et al., 2002; Beets et al., 2007; Martínez Ros, 1999; Pieron et al., 2010). Reconociendo la importancia de este enfoque, este artículo busca analizar el problema desde una perspectiva más amplia que permita alcanzar un triple objetivo. En primer lugar, proponer el ocio como un punto de vista innovador desde el que entender e intervenir en los problemas de adherencia deportiva que experimentan los jóvenes actualmente; y en segundo lugar, proponer los itinerarios de ocio como concepto científico y herramienta de análisis adecuado para conocer los factores que favorecer la continuidad de la práctica deportiva juvenil (adherencia deportiva).

Los itinerarios de ocio constituyen el marco epistemológico idóneo para abordar la naturaleza psicosocial del ocio y su carácter procesual, como fenómeno que acompaña a la persona a lo largo de su existencia. Desde el enfoque del ciclo vital y, apoyados en las corrientes teóricas que han profundizado en el estudio del ocio a lo largo de la vida se plantean aquí cuestiones acerca de la evolución del ocio deportivo, los patrones de cambio que subyacen a este proceso, la posibilidad de comprender a las personas en función de tales cambios, la capacidad predictiva de estos conocimientos y su valor a la hora de favorecer la persistencia del ocio deportivo a lo largo de la vida e impulsar su papel como factor de desarrollo personal. En definitiva, la incorporación del concepto itinerario al estudio del ocio a lo largo de la vida aporta nueva luz a estas cuestiones y facilitará la intervención para evitar estilos de ocio sedentarios y decisiones de abandono definitivo de la práctica deportiva entre los jóvenes.

El ocio deportivo en clave de experiencia y su contribución al desarrollo humano

Lejos queda la comprensión del ocio como un fenómeno social negativo. Igualmente lejos quedan las aproximaciones a su estudio entendido como tiempo libre o actividad. Hace ya décadas que esta forma de entender el ocio resulta insuficiente puesto que sólo permite una comprensión limitada de este fenómeno. Aceptar que el ocio es sinónimo de tiempo libre o de actividad es olvidar el conjunto de variables que toman parte en las conductas de ocio (cogniciones, emociones, motivaciones, valores...) así como las experiencias personales y colectivas que viven sus protagonistas. Los avances habidos en el conocimiento del 
ocio plantean la necesidad de comprender este fenómeno en clave de experiencia (Csikszentmihalyi, 1997, 2000; Cuenca, 2006, 2010; Lewis, 2000; Tinsley \& Tinsley, 1986). La importancia de la experiencia de ocio se encuentra en el alto valor que la persona le atribuye y en el hecho de que la actividad realizada se convierte en un fin en sí mismo que no necesita de otros argumentos para su justificación (Monteagudo, 2008). La importancia de la dimensión subjetiva del ocio no anula, sin embargo, su naturaleza social, fuertemente condicionada por las circunstancias del contexto social, económico, político o religioso en el que el ocio tiene lugar.

Este reconocimiento del ocio como experiencia que puede contribuir al desarrollo humano (Cuenca, 2006, 2010; Cuenca Amigo, 2013; Frey, 2012; Kleiber et al., 2011; Lazcano, Madariaga \& Doistua, 2010; Nussbaum, 2012; San Salvador del Valle, 2008) se extiende también al deporte por ser éste uno de los ámbitos en los que el ocio se manifiesta. Entender la práctica deportiva como una experiencia de ocio supone entenderla como una actividad libre, deseada e intrínsecamente motivada. Una actividad en la que la persona se encuentra consigo misma y con los demás; con la que puede adquiere destrezas y habilidades deportivas, superar los retos que se propone y desarrollar valores tales como la perseverancia, compromiso y respeto a los demás. Junto a los numerosos beneficios relacionados con la salud que la actividad deportiva puede proporcionar, encontramos que la práctica deportiva vivida como ocio puede ser fuente de beneficios cognitivos, emocionales, relacionales, etc. (Bailey et al., 2005; Fraguela et al., 2009; Monteagudo, 2008). En su conjunto y convenientemente planteado como proceso, el ocio deportivo puede favorecer el crecimiento personal y social por su capacidad para transformar positivamente a quien lo protagoniza. De ahí que la renuncia a este potencial ámbito de desarrollo humano nunca debería producirse por falta de oportunidades para vivir la práctica deportiva como experiencia de ocio.

Ahora bien, es importante subrayar que no todas las experiencias deportivas dejan la misma huella en el individuo ni contribuyen de la misma manera a su desarrollo personal. Tradicionalmente, las investigaciones sobre los hábitos deportivos de los ciudadanos han puesto de relieve el protagonismo de la dimensión lúdica del ocio deportivo. La identificación del deseo de divertirse, entretenerse y pasarlo bien es uno de los motivos más extendidos para hacer deporte (García Ferrando, 2006; López Ruiz, 2006; Sanz, 2005). Sin embargo, suponer que la diversión que proporciona la práctica deportiva es la única aportación que el ocio deportivo puede hacer al desarrollo humano es un grave error. Las vivencias deportivas en las que predomina el entretenimiento como mera forma de dar contenido al tiempo libre y en las que se adopta una actitud pasiva o escasa implicación son una manera de vivir el ocio pero no la única (Csikszentmihalyi, 2000). Hay que saber el grado de implicación con la práctica correlaciona positivamente con la satisfacción que ésta propicia. De ahí que un ocio que requiere dedicación, compromiso, esfuerzo, constancia y orientación a la superación ofrece mayores posibilidades de ser un ocio satisfactorio, al ser también un ocio más complejo.

En la práctica deportiva como en otras expresiones de ocio, la permanencia en el nivel de la diversión sitúa la experiencia en el terreno de un ocio casual, caracterizado por la ausencia de esfuerzo, el logro inmediato de satisfacción y el carácter puntual de la acción realizada. Por el contrario, la búsqueda progresiva de otros elementos que enriquezcan la experiencia deportiva desemboca en expresiones de ocio íntimamente relacionadas con experiencias óptimas o de flujo y con procesos de autorrealización y desarrollo personal (Csikszentmihalyi, 2000; Stebbins, 2000a, 2005).

En consecuencia, existen diferentes maneras de vivir la práctica deportiva entendida como ocio (Figura 1); todas ellas legítimas e interesantes para el bienestar y la calidad de vida. Ahora bien, para disfrutar de un ocio deportivo duradero a lo largo de la vida y promotor de desarrollo, parece necesario no limitarse a vivir la práctica deportiva únicamente como fuente de diversión sino que conviene incorporar progresivamente elementos que aumenten el grado de complejidad de la experiencia deportiva, garantizando así la continuidad del interés y el apego emocional del protagonista hacia la práctica que realiza.

\section{El Ocio Deportivo como Proceso: Aportaciones desde el Concepto de Itinerario}

Los avances realizados por las corrientes teóricas que abordaban el estudio del ocio desde los presupuestos del Ciclo vital han sido claves para comprender que ni el ocio ni la práctica deportiva deben limitarse a una etapa concreta de la vida sino que han de acompañar a la persona durante toda la vida (Armstrong \& Morgan, 1998; Atchley, 1989; Cuenca, 2006; Iso-Ahola, 1980; Kleiber et al., 2011; Nimrod \& Kleiber, 2007).

El estudio de los cambios que el ocio experimenta durante el ciclo vital así como la identificación de tendencias que dominen la aparición de tales cambios ha generado un enorme interés en la comunidad científica y, al mismo tiempo, ha sido objeto de importantes controversias. Las Teorías del Desarrollo y en especial, la Teoría del Desarrollo del Ocio, elaborada por Iso-Ahola (1980) ha revelado que la evolución del ocio a lo largo de la vida se halla condicionada por la orientación dual de las personas hacia la estabilidad y 

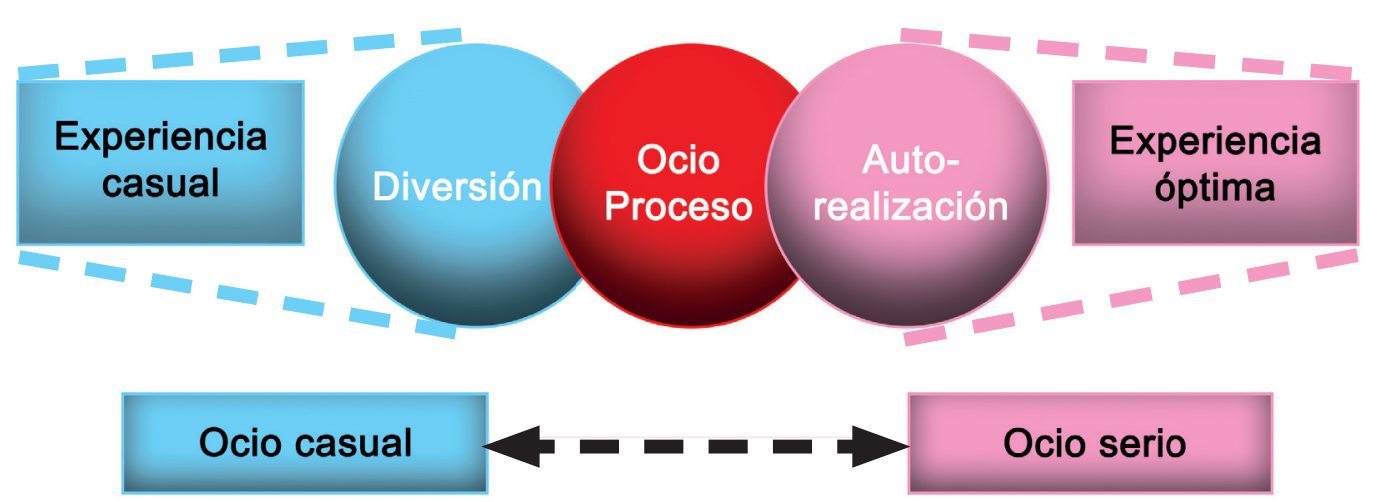

Figura 1. Formas de Aproximación a la Práctica Deportiva, Según la Complejidad de la Experiencia

el cambio. Las decisiones de ocio que tomamos para iniciar, continuar, sustituir o abandonar una práctica responden a la constante búsqueda de equilibrio entre estas dos tendencias que conviven en el ser humano.

A pesar de esta dualidad, encontramos numerosas investigaciones que tras analizar la evolución de las prácticas de ocio de población adulta y mayor hallan en sus resultados datos suficientes para apoyar una tendencia a la continuidad en los patrones de ocio (Armstrong \& Morgan, 1998; IsoAhola, Jackson, \& Dunn, 1994; Iwasaki \& Smale, 1998; Jackson \& Dunn, 1988; Lee \& King, 2003; Searle, Mactavish $\&$ Brayley, 1993). Estos autores que han profundizado en esta dialéctica continuidad-cambio concluyen que existe en las personas una fuerte tendencia a buscar la repetición de aquellas actividades que nos han resultado satisfactorias. Sus hallazgos sugieren que más de la mitad de las actividades de ocio de una persona adulta tienen su origen en preferencias que se han creado durante la infancia (Iso-Ahola, Jackson, \& Dunn, 1994; McGuire, Dottavio, \& O'Leary, 1987).

Si realmente esto es así, nos hallamos ante un dato con amplias repercusiones desde el punto de vista de las políticas, la gestión y la intervención en materia de ocio. Significaría que la historia de ocio ya vivida constituye un punto de referencia importante para descubrir cómo será la trayectoria de ocio en etapas posteriores de la vida. Por ello, el estudio de las trayectorias de ocio deportivo durante la infancia y juventud puede resultar crucial para prever el papel que la práctica deportiva puede tener en la historia posterior de ocio de cada persona.

Potenciar el ocio deportivo a lo largo de la vida implicaría orientar esfuerzos y recursos a exponer a las personas, durante su infancia y juventud, a un amplio abanico de actividades de ocio deportivo que les permitan vivir experiencias satisfactorias, sentando las bases actitudinales y motivacionales necesarias para que deseen retomar su participación en dichas prácticas en etapas posteriores de la vida. Se ha demostrado que los argumentos que recurren a la salud para impulsar la práctica deportiva de la ciudadanía son efectivos a corto plazo pero no resultan adecuados para favorecer la participación deportiva a largo plazo. Por el contrario, la vivencia de experiencias deportivas satisfactorias, sustentadas en estilos de motivación intrínseca (en los que existe un elevado interés por la acción en sí misma), resulta fundamental para favorecer la continuidad deportiva. De hecho, la vivencia satisfactoria de la práctica deportiva es el mejor aliado de la adherencia deportiva por el apego emocional que la persona experimenta con la actividad realizada. Si las ofertas deportivas dirigidas al público juvenil tuvieran en cuenta esta premisa así como los diferentes factores que afectan las decisiones de continuidad o abandono deportivo en el colectivo juvenil, la consolidación de estilos de ocio físicamente activos entre los jóvenes sería un objetivo más viable.

En este escenario, el uso del concepto itinerario de ocio deportivo resulta más que adecuado para analizar desde un punto de vista innovador el problema del sedentarismo y el abandono deportivo de la población juvenil actual.

Como se ha podido comprobar anteriormente, existen numerosas las investigaciones que desde la Psicología del Ocio y, apoyándose en las Teorías del Ciclo Vital, pretenden clarificar la evolución que el ocio experimentan a lo largo de la vida; sin embargo, no se ha localizado en la literatura revisada ninguna investigación que, de manera explícita, aplique este concepto de itinerario al fenómeno del ocio. El estudio de los itinerarios ha sido empleado, principalmente, en el ámbito de la Sociología del Deporte (Masnou \& Puig, 1995; Puig \& Masnou, 1988), razón por la cual, su incorporación a los Estudios de Ocio resulta ser algo nuevo en la trayectoria de esta disciplina.

Partiendo de la definición del concepto de Puig y Masnou (1995, p.371), se entiende por itinerario de ocio deportivo el conjunto de experiencias deportivas que una persona ha 
disfrutado a lo largo de su historia de ocio. Dicho itinerario se "inicia en el instante en que el sujeto toma contacto con el ocio deportivo; se desarrolla durante un periodo de tiempo más o menos amplio en el que se dedica a la práctica de una o varias actividades deportivas y finaliza cuando se abandona toda relación con este ámbito como participante". Habitualmente, las fases por las que un itinerario transcurre se representan a través de tres momentos bien diferenciados: el inicio, que corresponde al momento en que la persona inicia su relación con la actividad; el desarrollo, cuando la persona protagonista opta por dar continuidad o abandono temporal de algunas de sus prácticas; y finalmente, la resolución del itinerario, con la que la persona pone fin a su participación deportiva a través del abandono definitivo.

La relevancia de este concepto radica, por un lado, en la oportunidad que proporciona al investigador de entender el conjunto de las experiencias deportivas de una persona como un todo integrado con sentido que es su historia deportiva. Por otro, el potencial del concepto itinerario descansa en su capacidad predictiva; esto es, en la posibilidad que ofrece de identificar tendencias y patrones de cambio que convierten la historia de ocio deportivo ya vivida por cada persona en una base sólida para delinear el futuro de dichas trayectorias en cuanto a práctica deportiva se refiere. Desde el punto de vista de la investigación, el concepto de itinerario es una herramienta esencial para reconstruir las historias de ocio deportivo ya vividas y delimitar, a través de su estudio, los factores psicosociales responsables de las decisiones que las personas adoptan sobre su participación deportiva, optando por la continuidad o el abandono, sea éste temporal o definitivo.

Teniendo en cuenta todo lo anterior y conscientes de la necesidad de promover estilos de ocio deportivo duraderos desde las primeras etapas de la vida, se inicia una investigación que busca reconstruir, entre 1995 y 2005, las experiencias de ocio deportivo de una muestra de jóvenes españoles, residentes en Bizkaia, con el propósito de identificar sus itinerarios deportivos y los factores psicosociales que propician la continuidad de su participación deportiva. Más concretamente, esta investigación se plantea tres objetivos fundamentales:

- Conocer la situación de la práctica deportiva juvenil en Bizkaia entre 1995 y 2005, atendiendo a su presencia en los estilos de ocio juveniles y las formas de participación deportiva desarrolladas.

- Reconstruir las experiencias de ocio que la práctica deportiva ha propiciado a estos jóvenes, atendiendo a la evolución temporal de sus conductas deportivas.

- Evaluar el impacto que su entorno social ha tenido en sus historias deportivas, mediante el estudio de su entorno familiar, educativo, relacional y comunitario.

Esta investigación parte de la hipótesis de que la historia deportiva desarrollada durante etapas tempranas de la vida, esto es, infancia y juventud, ejercen un gran influjo en relación a la participación deportiva que ha de tener lugar en periodos posteriores. En consecuencia, la etapa juvenil brinda la posibilidad de analizar, con un enfoque retrospectivo, las historias deportivas de los jóvenes, identificar los tipos de itinerarios que éstas configuran y delimitar los factores personales y sociales que favorecen su participación deportiva.

\section{Método}

Esta investigación forma parte de un estudio más amplio solicitado en el año 2005 por las administraciones del País Vasco, en el norte de España y llevado a cabo por el Instituto de Estudios de Ocio de la Universidad de Deusto. De ahí que los principales resultados obtenidos así como las propuestas de acción con las que culmina el estudio tuvieran un valor estratégico por su contribución a la mejora de las políticas deportivas vascas. Centrada en el caso de Bizkaia, uno de los tres Territorios Históricos que conforman actualmente el País Vasco, la presente investigación describe con detalle el perfil de la muestra de jóvenes implicados, el instrumento de medida empleado para recabar la información necesaria y el procedimiento seguido para ello.

\section{Participantes}

El universo objeto de estudio de esta investigación lo conforma el conjunto de jóvenes de Bizkaia, de ambos géneros, residentes en cualquier municipio de este Territorio Histórico y nacidos en 1985; de forma que poseen 19 ó 20 años en el momento en que se lleva a cabo la investigación. La muestra la componen 383 jóvenes que, cumpliendo los criterios previamente indicados, fueron seleccionados entre la población total mediante un muestreo bietápico estratificado por municipios y cuotas de género. El tamaño muestral fue diseñado teniendo en cuenta los datos proporcionados por el Instituto Vasco de Estadística (EUSTAT) correspondientes al censo de población de 2001, con un nivel de confianza de $95 \%$ y un error muestral de $+/-5 \%$. La muestra está compuesta por $51,2 \%$ hombres y $48,8 \%$ mujeres, la mayoría $(87,7 \%)$, estudiantes con situaciones académicas y perfiles formativos diversos.

En consecuencia, el estudio empírico de esta tesis se lleva a cabo atendiendo a los criterios estadísticos establecidos y que se resumen en la siguiente ficha técnica: 


\begin{tabular}{ll}
\hline Universo & $\begin{array}{l}\text { Jóvenes de ambos géneros } \\
\text { residentes en Bizkaia y } \\
\text { nacidos en } 1985 .\end{array}$ \\
& $\begin{array}{l}\text { Territorio Histórico de } \\
\text { Bizkaia }\end{array}$ \\
Ámbito geográfico & 383 encuestas \\
Tamaño muestral & $+/-5 \%$ \\
Error muestral & $95 \% \mathrm{Z}=1,96 \mathrm{p}=\mathrm{q}=0,5$ \\
Nivel de confianza & Muestreo bietápico \\
& estratificado por municipios \\
Diseño muestral & y cuotas de género. \\
\hline
\end{tabular}

El análisis de las prácticas deportivas de los jóvenes vizcaínos de la muestra se llevó a cabo, tal y como se observa en la Tabla 1, mediante la reconstrucción de sus experiencias deportivas y la evolución de sus trayectorias, atendiendo al tipo de relación mantenida con la práctica deportiva entre 1995 y 2005: regular, es decir, con una frecuencia igual o superior a dos días a la semana y con una duración igual o superior a dos años consecutivos; esporádica, esto es, sin alcanzar los parámetros previamente considerados para la práctica regular; ausente, en el caso de aquellos jóvenes que hacen de la ausencia de práctica deportiva uno de los rasgos característicos de sus estilos de ocio.

Tabla 1

Aproximación a la Práctica Deportiva Entre 1995-2005

\begin{tabular}{lcc}
\hline & $\mathbf{N}$ & $\mathbf{\%}$ \\
\hline Regulares & 221 & $57,7 \%$ \\
Esporádicos & 82 & $21,4 \%$ \\
No practicantes & 80 & $20,9 \%$ \\
\hline Total & $\mathbf{3 8 3}$ & $\mathbf{1 0 0 , 0 \%}$ \\
\hline
\end{tabular}

Esta distinción y la segmentación de la población que conlleva en tres grupos bien diferenciados, resulta clave por varias razones. En primer lugar, tal y como se pone de relieve en la literatura científica revisada (COMPASS, 1999), porque la regularidad (frecuencia y continuidad) son algunas de las condiciones que estudios de calado internacional apuntan como fundamentales para lograr que la práctica deportiva constituya una fuente de salud y calidad de vida. En segundo lugar, y centrándonos en el marco teórico del ocio que preside este estudio, porque la dedicación regular a una actividad deportiva propicia una vivencia deportiva radicalmente distinta y que puede acercarse a la idea de ocio serio por la que abogan algunos teóricos del ocio (Stebbins, 2000a, 2005). En definitiva, los antecedentes encontrados en la literatura científica para la identificación de diferentes itinerarios deportivos apuntan a la práctica deportiva regular como elemento de referencia para el análisis planteado.

\section{Instrumento}

La presente investigación se apoya en un cuestionario elaborado ad hoc para este estudio. El diseño y contenidos del cuestionario buscan aportar la información necesaria para describir y comprender la presencia y relación que los jóvenes de la muestra han mantenido con la práctica deportiva entre 1995 y 2005, desentrañando el sentido y naturaleza de sus experiencias de ocio, la posible existencia de un patrón que defina sus trayectorias deportivas en términos de itinerario y los factores psicosociales que condicionan su participación deportiva /regular, esporádica o ausente) así como sus decisiones de continuidad o abandono deportivo.

Para su elaboración se ha tenido en cuenta otros cuestionarios y herramientas de naturaleza cuantitativa, bien considerados por la comunidad científica vinculada a los temas tratados. Cabe mencionar entre las más significativas, la encuesta quinquenal realizada por el Consejo Superior de Deportes para la elaboración del estudio sobre hábitos deportivos de la población española. Esta herramienta es un referente ineludible en el contexto español. Concretamente, los ítems que han servido de referencia y apoyo en esta tesis han sido los correspondientes a la encuesta realizada en 2005, disponible en la obra Posmodernidad y Deporte: entre la individualización y la masificación. Encuesta sobre hábitos deportivos de los españoles 2005 (García Ferrando, 2006). Igualmente, la herramienta empleada por el equipo de investigación de Emilia Fernández y colaboradores para el estudio realizado en 2004 sobre Evolución de la práctica de la actividad física y el deporte en mujeres adolescentes e influencia en la percepción del estado general de salud (Martínez de Quel Pérez, Fernández, \& Camacho, 2010) y la Encuesta de hábitos deportivos en la CAPV (Gobierno Vasco, 2009).

El cuestionario definitivo, Cuestionario para la identificación de los itinerarios deportivos juveniles, factores promotores de la práctica deportiva regular y su continuidad, contempla las siguientes secciones con sus categorías de análisis y variables correspondientes:

- Variables sociodemográficas o de identificación (8 items)

- Resumen Relación con la Práctica Deportiva 19952005 (8 items)

- Práctica Deportiva Regular (23 items)

- Práctica Deportiva Esporádica (3 items)

- Ausencia de Práctica Deportiva (3 items)

- Valoración personal del Entorno Social (Familiar, 
Educativo, Relacional y Comunitario) (32 items)

\section{Procedimiento}

Una vez elaborada la herramienta, se llevó a cabo un pre-test de 25 encuestas, con resultados positivos que permitieron iniciar la administración del cuestionario definitivo de carácter anónimo. El trabajo de campo se inicia en mayo de 2005 y se extiende durante los tres meses siguientes hasta finales de julio de este mismo año. La administración de los 383 cuestionarios fue realizada por un equipo de encuestadores, previamente formados mediante sesiones específicas, cuyo objetivo era darles a conocer los objetivos del estudio, estructura y contenidos del cuestionario así como los procedimientos que debían tener en cuenta para acceder a la muestra seleccionada. Con el fin de facilitar la labor de los encuestadores y garantizar la rigurosidad y homogeneidad en la administración del cuestionario, se elaboró un material de apoyo que fue utilizado tanto en las sesiones formativas como durante el trabajo de campo. Se trata de un Manual de Administración del Cuestionario y de varias Tarjetas de Apoyo que, a modo de Anexo, acompañan al cuestionario. Estas tarjetas han sido diseñadas con el fin de agilizar la enunciación de cada item por parte del encuestador/a así como la identificación y codificación de las respuestas vinculadas a cada pregunta.

Tras el trabajo de campo y el correspondiente proceso de depuración de los cuestionarios se procedió a la explotación de los datos con el programa SPSS (versión 19.0). La aplicación de estadísticos descriptivos y de correlación permitió conjugar la información de conjunto, obtenida del total de los jóvenes de la muestra con información sectorial específica de cada grupo de jóvenes planteados en función de su relación con la práctica deportiva: regular, esporádica o ausente. De esta forma ha sido viable, en primer lugar, la identificación de los itinerarios de ocio deportivo en el colectivo de jóvenes con práctica regular por ser éstos los únicos con una trayectoria deportiva suficientemente sólida como para evidenciar distintos patrones derivados de la evolución de la participación deportiva habida entre 1995 y 2005; en segundo lugar, la detección de los factores que favorecen entre los jóvenes la dedicación regular a la práctica deportiva, mediante la comparación de las experiencias de ocio deportivo de los tres grupos de jóvenes identificados; y finalmente, la detección de los factores promotores de la continuidad de la práctica deportiva regular, a partir del análisis comparativo de los itinerarios deportivos identificados.

\section{Principales Resultados}

En este apartado se resumen los principales resultados obtenidos de este estudio estructurados en dos grandes apartados.

En el primero de ellos, dedicado a las Prácticas de ocio deportivo de los jóvenes analizados, se resumen los principales hallazgos en relación a tres cuestiones:

- Situación y características del ocio deportivos de los jóvenes de Bizkaia.

- Evolución temporal de sus prácticas entre 1995 y 2005.

- Entornos que han incidido en la configuración de sus trayectorias deportivas.

El segundo apartado, centra la atención en los itinerarios de ocio deportivo detectados entre los jóvenes de Bizkaia $\mathrm{y}$ en los factores personales y sociales implicados en las decisiones de continuidad o abandono que configuran dichos itinerarios. Dos son, por tanto, los puntos desarrollados a este respecto:

- Los itinerarios de ocio deportivos de los jóvenes de Bizkaia.

- Factores psicosociales implicados en la orientación hacia la Práctica Deportiva Regular (PDR) y su continuidad.

\section{Prácticas de Ocio Deportivo de los Jóvenes de Bizkaia}

Los datos obtenidos en relación a la participación deportiva actual de los jóvenes vizcaínos confirman las siguientes premisas:

\section{Situación actual y características del ocio deportivo juvenil en Bizkaia.}

La práctica deportiva ocupa un lugar privilegiado en el repertorio de ocio de los jóvenes vizcaínos desde la infancia $(79,1 \%)$. Durante el periodo analizado, prácticamente, 6 de cada 10 jóvenes vizcaínos $(57,7 \%)$ ha hecho deporte mediante un patrón regular, el 21,4\% de manera esporádica y el $20,9 \%$ se han mantenido ajenos a este ámbito del ocio. La práctica deportiva regular es, por tanto, la forma de hacer deporte más extendida entre los jóvenes vizcaínos. La aproximación o distanciamiento de los jóvenes a esta forma de hacer deporte, se muestra condicionada por el género, dado que sus protagonistas son mayoritariamente hombres $(66,3 \%)$, frente a una mayoría de mujeres en el grupo de practicantes esporádicos y no practicantes $(58,5 \%$ y $60 \%$, respectivamente). Se trata de jóvenes que inician su actividad deportiva a edades tempranas, principalmente antes de los 10 años (41,6\%). La incorporación tardía a la práctica deportiva regular es un hecho característico de las mujeres practicantes regulares (18/19 años). En este grupo prevalece la dedicación a una única actividad deportiva $(69,6 \%)$ y aunque aún persisten 
modalidades mayoritarias como fútbol o baloncesto, las prácticas regulares se mueven en torno a un amplio espectro de modalidades que combina deportes de corte tradicional (fútbol y baloncesto), con otras prácticas de talante más contemporáneo, cuya fuerza aumenta a medida que avanza la edad (fitness, surf, deportes de invierno...). Desde un enfoque de género, se evidencian las diferentes direcciones que siguen las elecciones deportivas de hombres y mujeres. Circunstancia que aún legitima la identificación de modalidades eminentemente masculinas o femeninas, sin perjuicio de otras modalidades que pueden definirse como neutras (baloncesto, balonmano, surf y deportes de invierno). La diversión es un elemento común de las prácticas deportivas regulares de los jóvenes, al margen del tipo de práctica realizada (deporte escolar, de rendimiento o participación); resulta clave en los procesos de iniciación, sin embargo, su consecución no garantiza la continuidad de las prácticas deportivas. Entre los motivos que los jóvenes practicantes esporádicos emplean para argumentar su alejamiento de la práctica regular, resalta su preocupación por los estudios (19,5\%) y el sentimiento de "pereza" (17,1\%). El uso de este argumento denota que la práctica deportiva no resulta suficientemente atractiva para este colectivo.

Evolución temporal de las prácticas deportivas juveniles entre 1995 y 2005.

El inicio temprano de la vida deportiva -preferentemente, durante la infancia-, favorece la dedicación regular a este ámbito y crea las condiciones adecuadas para su continuidad, pero no la garantiza puesto que un porcentaje importante de jóvenes que se inicia en la práctica deportiva regular antes de los 13 años, acaba abandonando su dedicación deportiva (36,5\%). Las decisiones de continuidad están asociadas a la satisfacción de la necesidad de competencia, a través de la percepción de mejora de habilidades que retroalimenta el interés intrínseco por la práctica realizada. El abandono deportivo es un rasgo instaurado en los estilos de ocio deportivo de los jóvenes vizcaínos practicantes regulares. No obstante, su importancia no se debe al hecho cuantitativo, sino a su impacto sobre la vida deportiva pues en buena parte de los casos $(48,2 \%)$, supone una ruptura definitiva con la dedicación regular al deporte. Durante los once años analizados, el 53,4\% de los jóvenes practicantes regulares ha interrumpido alguna de las actividades realizadas. El análisis longitudinal confirma que el abandono hace especial mella entre los 12 y los 16 años, y alcanza su máxima expresión a los 14 años. Esta concentración, ya corroborada en otros estudios (Cecchini, Méndez, \& Contreras, 2005; Fernández, 2007; Martínez de Quel et al., 2010), puede explicarse recurriendo a factores asociados al sistema educativo (paso entre niveles educativos) y también al deportivo, concretamente, al deporte escolar (cambios de categoría). Emergen como principales motivos de abandono, las responsabilidades académicas (17,40\%), las limitaciones de tiempo que conlleva la dedicación regular a la práctica deportiva $(15,20 \%)$, el aburrimiento y el cambio de centro de estudio (10,90\%, en ambos casos).

\section{Entornos que más han incidido en el desarrollo de las trayectorias deportivas juveniles.}

El entorno familiar, representando por los progenitores, y el entorno relacional, conformado por el grupo de iguales, ejercen un papel esencial en el desarrollo deportivo juvenil. El impacto de los progenitores en la participación deportiva se evidencia a través de la importancia otorgada a esta actividad, los valores que en ella destacan así como la percepción y naturaleza del apoyo que los progenitores proporcionan a sus hijos en materia deportiva. Se constata, en primer lugar, que la relevancia otorgada a la práctica deportiva por las familias es mayor, cuanto mayor es la implicación deportiva de los jóvenes; esto es, en el caso de la práctica deportiva regular. La diversión y los beneficios que la actividad deportiva proporciona son, por orden de importancia, los valores que las familias más destacan en el hecho deportivo. Esta afirmación es especialmente evidente entre los practicantes regulares. Parece existir cierta relación entre los valores del contexto familiar hacia la práctica deportiva y la participación real de los jóvenes en este ámbito. En segundo lugar, la percepción de apoyo familiar por parte de los jóvenes es también, un factor diferencial del tipo de interacción mantenida con la práctica deportiva. Dicha percepción es mayor, cuanto mayor es la dedicación a la práctica deportiva. Por tanto, los jóvenes practicantes regulares perciben más el apoyo de sus familias que el resto de los grupos (practicantes esporádicos y no practicantes). Además, prevalece entre los jóvenes practicantes regulares un apoyo familiar de tipo emocional que, tal y como se comprueba en la literatura, resulta el más efectivo, en términos de participación y adherencia deportiva (Duncan, Duncan, \& Strycker, 2005). El impacto de las amistades se revela en este estudio como un factor capaz de condicionar la participación deportiva juvenil. La naturaleza social de la acción deportiva emerge como una constante en los jóvenes de la muestra. Los jóvenes deportistas vizcaínos buscan amistades que consideran hacer deporte una actividad valiosa. Quienes hacen deporte de manera regular o esporádica admiten el influjo positivo de las amistades en su participación deportiva pues el hecho de rodearse de amistades que valoran y practican deporte, les anima a continuar practicando. Por el contrario, los pocos jóvenes sedentarios que admiten la incidencia de las amistades en su vida deportiva consideran que ésta ha sido negativa ya que el 
hecho de relacionarse con jóvenes sedentarios ha contribuido a su decisión de mantenerse alejado de este ámbito. Un argumento que ratifica de nuevo la capacidad de los iguales para promover o inhibir la participación deportiva.

Por el contrario, el análisis del entorno escolar, considerando la figura de los centros escolares, su posicionamiento ideológico ante la práctica deportiva y las condiciones que reúnen para promover esta actividad y el entorno comunitario (existencia o no de equipamientos, áreas deportivas recreativas y clubes deportivos en los municipios de residencia), no ejercen, en opinión de los propios jóvenes, un influjo relevante en su participación deportiva.

\section{Identificación de Itinerarios Deportivos Juveniles y Factores Implicados en su Evolución}

El desarrollo de las historias deportivas viene marcado por los cambios que éstas experimentan, cada vez que los jóvenes toman una decisión en materia deportiva. Dichos cambios resultan esenciales en el proceso de definición de los itinerarios deportivos, pues de ellos depende la dirección dominante en cada caso, así como la adhesión de cada joven a un determinado itinerario. Sin embargo, no todos los cambios que tienen lugar en las historias deportivas poseen la misma incidencia en la configuración de sus itinerarios. Los abandonos puntuales de actividad o la sustitución de una práctica por otra, primordiales en una visión "micro", centrada el devenir de cada una de las actividades que conforman las historias deportivas individuales, dejan paso ahora a una mirada más global; una visión "macro", cuyo interés radica exclusivamente en aquellos movimientos, susceptibles de modificar la dirección de las trayectorias desarrolladas hasta entonces. En este escenario, el abandono de una actividad regular no adquiere trascendencia si el contacto regular con este ámbito queda preservado a través de otra(s) práctica(s) que no se abandona(n). Tampoco un cambio de una actividad por otra, no ha de suponer una interrupción de la dedicación deportiva, si la nueva actividad se desarrolla en condiciones de regularidad y no media entre ambas un periodo de ausencia de práctica superior a un año. De acuerdo con este planteamiento, resulta esencial incorporar en este punto, el concepto de Vida deportiva Regular (VDR), por su capacidad para demarcar los diferentes hitos responsables del devenir de las historias deportivas de cada individuo. Entendemos por VDR, el o los periodos ininterrumpidos en los que cada joven mantiene una interacción regular con la práctica deportiva a través de una $o$ varias actividades. Este enfoque permite la distinción entre abandono temporal y definitivo, otorgando un significado distinto a las decisiones de abandono, en función de sus consecuencias en la trayectoria deportiva. De esta forma, este concepto explica que el abandono de una práctica no suponga la ruptura de la VDR si se continúa practicando regularmente otras actividades que ya se hacían de forma simultánea.

De acuerdo con esta visión más global del abandono, la identificación de los itinerarios deportivos de los jóvenes de Bizkaia se ha llevado a cabo mediante la agrupación de las historias deportivas juveniles, atendiendo a cuatro criterios: edad de inicio de la VDR entre los 10 y 20 años; existencia o ausencia de experiencias de abandono de la VDR; carácter temporal o permanente del abandono y, situación de la trayectoria deportiva en 2005 , año de finalización del estudio.

\section{Los itinerarios deportivos de los jóvenes de Bizkaia.}

Los itinerarios deportivos encontrados entre los jóvenes vizcaínos practicantes regulares son:

- Itinerario 1. Jóvenes Tempranos Continuistas. Jóvenes que inician VDR antes de los 13 años y no la abandonan, al menos, hasta el año 2005.

- Itinerario 2. Jóvenes Tempranos Rupturistas. Jóvenes que inician VDR antes de los 13 años, experimentan un abandono que rompe su contacto regular con este ámbito y no vuelven a recuperarlo.

- Itinerario 3. Jóvenes Tempranos Discontinuos Persistentes. Jóvenes que inician VDR antes de los 13 años, experimentan al menos un abandono de su VDR, pero vuelven a recuperarla y la mantienen hasta el año 2005.

- Itinerario 4. Jóvenes Tempranos Discontinuos Disuadidos. Inician VDR antes de los 13 años, experimentan el abandono de su VDR, vuelven a recuperarla pero abandonan de nuevo, antes de 2005.

- Itinerario 5. Jóvenes Intermedios Continuistas. Inician VDR entre los 13 y 16 años y no la abandonan, al menos, hasta el año 2005.

- Itinerario 6. Jóvenes Intermedios Rupturistas. Inician VDR entre los 13 y 16 años, pero deciden abandonar, interrumpiendo su contacto regular con este ámbito y no lo retoman.

- Itinerario 7. Jóvenes Tardíos Continuistas. Inician VDR entre los 17 y 20 años y no la abandonan, al menos, hasta 2005.

- Itinerario 8. Jóvenes Tardíos Rupturistas. Inician VDR entre los 17 y 20 años y experimentan antes de 2005 un abandono que anula su relación regular con la actividad deportiva.

De los 8 itinerarios identificados, únicamente tres resultan relevantes por aglutinar a la mayoría de los jóvenes: Itinerario $1(26,2 \%)$, Itinerario $2(35,2 \%)$ y Itinerario $7(20,3 \%) . \mathrm{Su}$ análisis sugiere que tres son los rasgos esenciales de los principales itinerarios deportivos de los 
jóvenes vizcaínos practicantes regulares:

- el comienzo temprano de la vida deportiva, especialmente durante la infancia (antes de los 13 años) aunque también los primeros años de juventud resultan propicios para el inicio; Los jóvenes integrantes del itinerario 7, identificados como tardíos continuistas son buena prueba de ello pues inician su VDR entre los 17 y 20 años.

- la tendencia a la continuidad de las prácticas deportivas, ilustrada a través de dos parámetros. El primero, la ausencia de abandono, que se erige como la característica dominante en dos de los tres itinerarios principales (itinerario 1 y 7); el segundo, la persistencia de las conductas deportivas regulares que llegan a alcanzar periodos de diez años de actividad ininterrumpida.

- la irrupción precoz del abandono de la $V D R$, que si bien es una realidad entre los jóvenes deportistas vizcaínos, no es un rasgo dominante entre los practicantes regulares de los itinerarios principales. Este fenómeno resulta especialmente preocupante por su irrupción en edades tempranas (entre los 12 y 14 años) y por sus consecuencias en el desarrollo deportivo de los jóvenes afectados. Hay que recordar que el alejamiento de la dedicación regular al deporte supone, la mayor parte de las veces, la no recuperación de las mismas condiciones de práctica. Tras el abandono, lo más habitual es la migración hacia la práctica deportiva esporádica; una tendencia que desciende, a favor de la no práctica, tanto más, cuanto más tarde tiene lugar el abandono.

Factores psicosociales implicados en la orientación hacia la práctica deportiva regular y su continuidad.

\section{Promotores de la Práctica Deportiva Regular (PDR).}

Resultado de la comparación de las experiencias deportivas de los tres colectivos de jóvenes analizados (regulares, esporádicos y no practicantes), se obtienen cinco factores que, avalados por los correspondientes resultados estadísticos, favorecen la dedicación regular a la práctica deportiva durante la etapa juvenil. Estos factores, explicados con más detalle a continuación, son:

1. Percepción de barreras

2. Edad de inicio de la vida deportiva

3. Género

4. Familia

5. Amistades

\section{Percepción de barreras.}

Cuanto mayor es la dedicación a la práctica deportiva, más relevante es la percepción de barreras estructurales y menor el peso de las barreras psicológicas o intrapersonales.
Los resultados revelan que el tipo de barreras percibidas para la práctica deportiva varía en función de la relación mantenida con este ámbito. La percepción de barreras psicológicas es más acusada entre los jóvenes sedentarios $(48,7 \%)$ y practicantes esporádicos $(32,9 \%)$, mientras que este tipo de barreras no resulta tan relevante entre los practicantes regulares $(15,2 \%)$. Es importante recordar que las barreras de carácter psicológico mantienen una correlación inversa con la motivación intrínseca y han sido identificadas en la literatura especializada como las variables más potentes para la predicción del compromiso con la práctica deportiva, debido al influjo que la percepción de este tipo obstáculos ejerce sobre los procesos motivacionales (Carroll, \& Alexandris, 1997). Según Crawford y Godbey (1987), las barreras psicológicas interfieren más en la formación de preferencias, que entre la preferencia ya formada y la toma de decisiones sobre la participación. Este hecho explica que la falta de interés, el sentimiento de pereza o el atractivo que ejercen otras alternativas de ocio sean algunos de los principales obstáculos que afectan a los jóvenes sedentarios y practicantes esporádicos. Todos ellos denotan la existencia de una escasa motivación intrínseca hacia la actividad deportiva entre jóvenes no practicantes y, en menor medida, entre los esporádicos, que no se evidencia entre los practicantes regulares.

\section{Edad de inicio.}

El inicio de la vida deportiva durante los años de infancia y juventud favorece la dedicación regular al deporte. La existencia de una relación estadísticamente significativa entre la edad de inicio de la vida deportiva y la práctica regular confirma que el inicio temprano de la vida deportiva constituye un factor promotor de la dedicación regular al ámbito deportivo, tal y como se había demostrado en estudios previos (Palau et al, 2005; Sanz, 2005; Ramos, Sanz, Valdemoros, \& Ponce de León, 2010). La Tabla 2 confirma que en esta investigación destacan especialmente, dos etapas: el periodo anterior a los 13 años y la franja de edad entre los 17 y 20 años. Esta última resulta especialmente propicia para el comienzo de la VDR entre las mujeres, ya que éstas se incorporan a este ámbito de manera más tardía que los hombres. El 29,7\% de las mujeres vizcaínas estudiadas inician su vida deportiva regular entre los 17 y 20 años, frente al $14,5 \%$ de los hombres.

A la luz de los resultados obtenidos parece necesario revisar el significado del término "temprano" pues la visión longitudinal que aporta el concepto de itinerario deportivo revela que, en el marco del ciclo vital, los comienzos de la participación deportiva tanto durante la infancia como durante 
Tabla 2

Edad de Inicio: Incidencia Para Hacer Deporte Regularmente

\begin{tabular}{lcccccc}
\hline $\mathbf{X 2 = 4 0 , 7 8 ;}$ sig=0,00 & \multicolumn{2}{c}{ Practicantes Regulares } & Practicantes Esporádicos & \multicolumn{2}{c}{ Total } \\
\hline Hasta 12 años & 152 & $74,5 \%$ & 52 & $25,5 \%$ & 204 & $100,0 \%$ \\
De 13 a 16 años & 22 & $59,5 \%$ & 15 & $40,5 \%$ & 37 & $100,0 \%$ \\
De 17 a 20 años & 47 & $79,7 \%$ & 12 & $20,3 \%$ & 59 & $100,0 \%$ \\
Ns/Nc & 0 & $0,0 \%$ & 3 & $100,0 \%$ & 3 & $100,0 \%$ \\
\hline Total & 221 & $27,1 \%$ & 82 & $72,9 \%$ & 303 & $100,0 \%$ \\
\hline
\end{tabular}

la juventud deben concebirse como inicios tempranos, ambos favorecedores de la dedicación regular a este ámbito.

\section{Género.}

No se puede afirmar con rotundidad que ser hombre es sinónimo de regularidad en la práctica deportiva, pero lo cierto es que el género masculino se halla más próximo a la participación regular que las mujeres, mejor representadas en los grupos de práctica esporádica o ausencia de práctica. Existe, tal y como se comprueba en la Tabla 3, una relación estadísticamente significativa que avala el impacto del género en la orientación hacia la VDR.

Se confirma, por tanto, el influjo bidireccional del género en la orientación hacia la práctica deportiva regular; ser hombre, se convierte en un factor facilitador de la VDR, mientras que ser mujer se torna un obstáculo o factor inhibidor para la práctica deportiva regular. El ámbito deportivo se muestra aún como un contexto en el que se reproducen con fidelidad los mecanismos sociales responsables de la construcción de diferencias entre géneros (Cecchini, Méndez, \& Contreras, 2005). La persistencia de tales mecanismos contribuye a explicar las divergencias encontradas en las experiencias deportivas de los hombres y mujeres de la muestra, tanto en relación a las modalidades deportivas realizadas, como en cuanto a la edad de inicio, la frecuencia de sus prácticas o la naturaleza de las barreras percibidas. Diferencias previamente constatadas en otros estudios tanto españoles como internacionales (Cavill et al., 2001; Fernández, 2007; García Ferrando, 2006; Instituto de la Mujer, 2006). Queda pendiente determinar si, una vez

Tabla 3

Género: Incidencia Para Hacer Deporte Regularmente

\begin{tabular}{|c|c|c|c|c|c|c|}
\hline $\mathrm{X} 2=12,26 ;$ sig $=0,00$ & \multicolumn{2}{|c|}{ Hombre } & \multicolumn{2}{|c|}{ Mujer } & \multicolumn{2}{|c|}{ Total } \\
\hline Regularmente & 130 & $58,8 \%$ & 91 & $41,2 \%$ & 221 & $100,0 \%$ \\
\hline Esporádicamente & 34 & $41,5 \%$ & 48 & $58,5 \%$ & 82 & $100,0 \%$ \\
\hline No practica & 32 & $40,0 \%$ & 48 & $60,0 \%$ & 80 & $100,0 \%$ \\
\hline Total & 196 & $51,2 \%$ & 187 & $48,8 \%$ & 383 & $100,0 \%$ \\
\hline
\end{tabular}

instaurada la práctica deportiva regular, el género ejerce también su impacto en la tendencia hacia la adherencia deportiva.

\section{Familia.}

Las relaciones estadísticamente significativas encontradas entre la práctica deportiva regular y el entorno familiar ratifican el papel de la familia como uno de los agentes implicados en la orientación de los jóvenes hacia la práctica deportiva regular. En general, la opinión acerca de la influencia positiva que el entorno familiar ha tenido en sus trayectorias deportivas está más extendida entre los jóvenes regulares (68\%). Practicantes esporádicos y no practicantes declaran que sus familias no han influido en su desarrollo deportivo de ninguna manera (50\%, en ambos casos). Más concretamente, los resultados obtenidos a este respecto permiten afirmar que, en primer lugar, la importancia concedida por el entorno familiar a la práctica deportiva es mayor cuanto más estrecha ha sido la relación del joven con la actividad deportiva; en segundo lugar, la diversión que proporciona la práctica deportiva es el aspecto más valorado por las familias de todos los jóvenes de la muestra, pero este reconocimiento es mayor en las familias de los practicantes regulares; en tercer lugar, el apoyo que los jóvenes de la muestra perciben de sus familias es menor, cuanto menor es el contacto con la práctica deportiva; y finalmente, el apoyo emocional es percibido de forma más habitual entre los 
practicantes regulares $(47,1 \%)$; los practicantes esporádicos reciben más apoyo informacional $(43,1 \%)$, mientras que los jóvenes que no han hecho deporte declaran no percibir ninguno.

\section{Amistades.}

Tal y como se confirma en investigaciones previas (Ponseti et al., 2005; Hernández et al., 2006; Ramos, Sanz, Valdemoros, \& Ponce de León, 2010), el círculo de amistades es otro de los factores implicados en el inicio de la vida deportiva regular. Los resultados obtenidos en relación a este entorno permiten argumentar la incidencia del entorno relacional en la decisión de practicar deporte de manera regular. Entre los principales hallazgos al respecto destacamos que la importancia concedida a la práctica deportiva por parte de las amistades del joven es mayor entre los practicantes regulares; compartir la práctica deportiva con las amistades es un hecho más frecuente entre los practicantes regulares; el reconocimiento de la influencia de las amistades en la práctica deportiva realizada es mayor entre los practicantes regulares; la práctica deportiva de las amistades constituye un refuerzo para el desarrollo deportivo de los jóvenes vizcaínos practicantes, tanto regulares como esporádicos.

Se constata así que la orientación hacia la práctica deportiva regular no depende únicamente de la predisposición personal hacia este ámbito. El interés decidido y la motivación intrínseca que requiere esta forma de hacer deporte, pueden consolidarse mediante la intervención de agentes sociales significativos que, según los resultados obtenidos son principalmente, familia y amistades. Ambos poseen un papel crucial en el desarrollo de actitudes positivas y el cultivo de la afición regular hacia el ocio deportivo. Su poder reside en su capacidad para actuar como promotores o detractores de la práctica deportiva regular.

\section{Promotores de la Continuidad de la Práctica Deportiva Regular}

Resultado de la comparación de los tres itinerarios deportivos juveniles más relevantes, se obtienen cinco factores que actúan como promotores de la continuidad de la práctica deportiva regular:

1. Concentración en actividad desarrollada

2. Búsqueda de objetivos, más allá del entretenimiento

3. Sentimiento de competencia

4. Percepción de inversión personal

5. Autonomía de las amistades

\section{Concentración en la actividad desarrollada.}

La concentración en el desarrollo de la práctica deportiva regular, sin que haya dedicación a actividad esporádica alguna, favorece la continuidad de la dedicación regular a la práctica deportiva. La Tabla 4 revela que este rasgo es una de las claves de los itinerarios continuistas y, se hace especialmente evidente en el itinerario 1, referente primordial de la continuidad de la vida deportiva.

Búsqueda de objetivos complejos más allá del entretenimiento.

A pesar de la hegemonía de la diversión como objetivo prioritario de los jóvenes de todos los itinerarios, resulta significativo comprobar en la Tabla 5 que esta búsqueda es menor entre los jóvenes que nunca abandonan la VDR (Itinerarios 1, 5 y 7). Centrando la atención en los jóvenes del itinerario 1, se observa en esta misma tabla que el deseo de pasarlo bien comparte protagonismo con otros objetivos, asociados a la búsqueda de resultados deportivos o de aprendizaje, en mayor medida que entre los jóvenes adheridos al itinerario 2, prototipo de abandono de la VDR. El hecho de que estos objetivos, más elaborados y exigentes, mediante los que se adivina el deseo de la persona de avanzar y crecer a través de la práctica deportiva, se asocien con la persistencia de las conductas deportivas, refuerza la afirmación de que el fenómeno del ocio va más allá de la mera diversión. Desde la Teoría del Ocio Humanista (Cuenca, 2006) defendida en este estudio se subraya la importancia de las experiencias de ocio como expresiones de desarrollo personal, en las que el deseo de superación y la percepción de competencia se convierten en las principales fuentes de motivación intrínseca y por tanto, en elementos promotores de continuidad.

La búsqueda de objetivos elaborados puede ser la expresión de formas más complejas de vivir la experiencia deportiva. Estas experiencias son importantes fuentes de satisfacción que retroalimentan el interés en la práctica desarrollada, contribuyendo así a explicar la persistencia de las conductas deportivas (Kleiber, 1999; Stebbins, 2000b). Igualmente, el protagonismo de la diversión entre los jóvenes del itinerario 2, sugiere que, por sí sólo, este objetivo, no garantiza la persistencia de las conductas deportivas a largo plazo. En la práctica deportiva son necesarios otros elementos que alimenten la motivación intrínseca y alienten la continuidad de la participación.

\section{Sentimiento de competencia como principal motivo de continuidad.}

La Tabla 6 evidencia que el deseo de "pasarlo bien" que incita el inicio de la VDR, es también el principal motivo de continuidad en casi todos los itinerarios. Un dato interesante porque revela la necesidad de dar respuesta a los motivos inicialmente planteados, como garantía de continuidad de la práctica deportiva, pero que, sin embargo, no discrimina entre los itinerarios deportivos encontrados. 
Por el contrario, la mejora de las habilidades deportivas constituye un motivo de continuidad en el que divergen los integrantes de los itinerarios continuistas y rupturistas. Son los jóvenes continuistas del itinerario 1 quienes arguyen, principalmente, la mejora de habilidades, como uno de los aspectos fundamentales que les anima a continuar. La consecución de dicha mejora convierte la actividad deportiva de estos jóvenes en una experiencia satisfactoria al haber alcanzado con ella, los fines perseguidos.

Estos resultados evidencian en primer lugar, que la continuidad depende, en buena medida, del logro de los motivos considerados en el inicio de la VDR; en segundo lugar que, el logro de los motivos iniciales, cuya ausencia puede desembocar en decisiones de abandono, no resulta suficiente sin embargo, para garantizar la adherencia a la práctica deportiva regular. El deseo de dar continuidad a la VDR viene impulsado por la presencia de otros motivos que sumergen al joven en formas superiores de desarrollo deportivo, a través de la mejora de habilidades, como expresión de las competencias adquiridas y los retos alcanzados. El dominio deportivo, que se busca hacer patente ante uno mismo y los otros significativos, propicia sentimientos de satisfacción derivados de una percepción de creciente competencia que reafirma el interés por la práctica deportiva y contribuye, al mismo tiempo, a establecer nuevos retos que preservan el deseo de continuidad.

Percepción de inversión personal a partir de largos periodos de práctica deportiva ininterrumpida.

Las elevadas medias correspondientes a los periodos de VDR asociadas a buena parte de los itinerarios encontrados son el principal indicador de la adherencia de los jóvenes regulares a la práctica deportiva. Lógicamente, las medias correspondientes a los periodos más largos de VDR, se asocian a itinerarios continuistas, en los que se aglutinan los jóvenes que no abandonan su actividad entre 1995 y 2005: itinerario 1 (10 años) y 5 (6 años). Ambos, son periodos que superan con creces el tiempo mínimo establecido en este estudio para identificar como regular una dedicación deportiva (al menos, dos años consecutivos de práctica).

Autonomía de las amistades en la toma de decisiones sobre la persistencia de las propias conductas deportivas.

Del total de los entornos analizados (entorno familiar, educativo relacional y comunitario), únicamente la familia y las amistades han ejercido, en opinión de los jóvenes practicantes regulares, un influjo positivo en su posterior desarrollo deportivo. A pesar su relevancia, el apoyo percibido por parte de la familia no discrimina en términos de continuidad o abandono, ya que es una constante entre los jóvenes de todos los itinerarios deportivos. Las divergencias emergen, exclusivamente, en relación a las amistades (Tabla 7). Éstas desempeñan un papel fundamental para los jóvenes de todos los itinerarios, excepto para los integrantes del itinerario 1 , al ser los únicos que declaran que sus amistades no han influido en su desarrollo deportivo $(62 \%)$. Esta opinión abre una importante brecha que escinde el itinerario 1 de la tendencia general en cuanto a las amistades e incluso, de los demás itinerarios continuistas ( 5 y 7). Los jóvenes continuistas del itinerario 1 no supeditan sus conductas deportivas a las decisiones de su círculo de amistades en este ámbito, haciendo gala de una independencia que vuelve a remarcar la orientación intrínseca de estos jóvenes

Tabla 4

Ausencia de PDE Como Promotor de la Continuidad de la PDR

\begin{tabular}{lcccccc}
\hline $\mathbf{X 2 = 4 0 , 7 8 ;}$ sig=0,00 & \multicolumn{2}{c}{ Práctica esporádica } & \multicolumn{2}{c}{ No práctica esporádica } & \multicolumn{2}{c}{ Total } \\
\hline Itinerario 1 & 13 & $22,4 \%$ & 45 & $77,6 \%$ & 58 & $100,0 \%$ \\
Itinerario 2 & 54 & $69,2 \%$ & 24 & $30,8 \%$ & 78 & $100,0 \%$ \\
Itinerario 3 & 11 & $84,6 \%$ & 2 & $15,4 \%$ & 13 & $100,0 \%$ \\
Itinerario 4 & 3 & $100,0 \%$ & 0 & $0,0 \%$ & 3 & $100,0 \%$ \\
Itinerario 5 & 3 & $37,5 \%$ & 5 & $62,5 \%$ & 8 & $100,0 \%$ \\
Itinerario 6 & 8 & $57,1 \%$ & 6 & $42,9 \%$ & 14 & $100,0 \%$ \\
Itinerario 7 & 22 & $48,9 \%$ & 23 & $51,1 \%$ & 45 & $100,0 \%$ \\
Itinerario 8 & 2 & $100,0 \%$ & 0 & $0,0 \%$ & 2 & $100,0 \%$ \\
\hline Total & 116 & $52,5 \%$ & 105 & $47,5 \%$ & 221 & $100,0 \%$ \\
\hline
\end{tabular}


Tabla 5

Orientación a Objetivos Más Allá de la Diversión, Factor de Continuidad de la PDR

\begin{tabular}{|c|c|c|c|c|c|c|c|c|c|c|c|c|}
\hline \multirow[b]{2}{*}{$X 2=58,26 ; \operatorname{sig}=0,01$} & \multicolumn{2}{|c|}{ Aprendizaje } & \multicolumn{2}{|c|}{ Rendimiento } & \multicolumn{2}{|c|}{ Diversión } & \multicolumn{2}{|c|}{$\mathrm{NS} / \mathrm{NC}$} & \multicolumn{2}{|c|}{ Varios } & \multicolumn{2}{|c|}{ Total } \\
\hline & $\mathrm{N}$ & $\%$ & $\mathrm{~N}$ & $\%$ & $\mathrm{~N}$ & $\%$ & $\bar{N}$ & $\%$ & $\mathrm{~N}$ & $\%$ & $\mathrm{~N}$ & $\%$ \\
\hline Itinerario 1 & 8 & $13,8 \%$ & 13 & $22,4 \%$ & 32 & $55,2 \%$ & 0 & $0,0 \%$ & 5 & $8,6 \%$ & 58 & $100,0 \%$ \\
\hline Itinerario 2 & 11 & $14,1 \%$ & 9 & $11,5 \%$ & 57 & $73,1 \%$ & 1 & $1,3 \%$ & 0 & $0,0 \%$ & 78 & $100,0 \%$ \\
\hline Itinerario 3 & 2 & $15,4 \%$ & 0 & $0,0 \%$ & 10 & $76,9 \%$ & 0 & $0,0 \%$ & 1 & $7,70 \%$ & 13 & $100,0 \%$ \\
\hline Itinerario 4 & 0 & $0,0 \%$ & 0 & $0,0 \%$ & 2 & $66,7 \%$ & 0 & $0,0 \%$ & 1 & $33,3 \%$ & 3 & $100,0 \%$ \\
\hline Itinerario 5 & 1 & $12,5 \%$ & 1 & $12,5 \%$ & 4 & $50,0 \%$ & 0 & $0,0 \%$ & 2 & $25,0 \%$ & 8 & $100,0 \%$ \\
\hline Itinerario 6 & 0 & $0,0 \%$ & 4 & $28,6 \%$ & 10 & $71,4 \%$ & 0 & $0,0 \%$ & 0 & $0,0 \%$ & 14 & $100,0 \%$ \\
\hline Itinerario 7 & 0 & $0,0 \%$ & 15 & $33,3 \%$ & 26 & $57,8 \%$ & 3 & $6,7 \%$ & 1 & $2,2 \%$ & 45 & $100,0 \%$ \\
\hline Itinerario 8 & 0 & $0,0 \%$ & 2 & $100,0 \%$ & 0 & $0,0 \%$ & 0 & $0,0 \%$ & 0 & $0,0 \%$ & 2 & $100,0 \%$ \\
\hline Total & 22 & $10,0 \%$ & 44 & $19,9 \%$ & 141 & $63,8 \%$ & 4 & $1,8 \%$ & 10 & $4,5 \%$ & 221 & $100,0 \%$ \\
\hline
\end{tabular}

Tabla 6

Percepción de Competencia, Factor Promotor de la Continuidad de la PDR

\begin{tabular}{lccllllllllll}
\hline X2=162,50; & Mejora \\
sig=0,00 & $\begin{array}{l}\text { Lo } \\
\text { halidada }\end{array}$ & $\begin{array}{l}\text { pasaba } \\
\text { muy } \\
\text { bien }\end{array}$ & $\begin{array}{l}\text { estaba } \\
\text { con } \\
\text { amigos }\end{array}$ & $\begin{array}{l}\text { Mi } \\
\text { familia } \\
\text { quería }\end{array}$ & $\begin{array}{l}\text { Me } \\
\text { sentía } \\
\text { popular }\end{array}$ & $\begin{array}{l}\text { Porque } \\
\text { ganaba }\end{array}$ & $\begin{array}{l}\text { Me } \\
\text { gustaba }\end{array}$ & $\begin{array}{l}\text { Quería } \\
\text { seguir }\end{array}$ & Otros & NS/NC & Varios & Total \\
Itinerarios & $\%$ & $\%$ & $\%$ & $\%$ & $\%$ & $\%$ & $\%$ & $\%$ & $\%$ & $\%$ & $\%$ & $\%$ \\
\hline I.1 & 31,0 & 46,6 & 6,9 & 0,0 & 0,0 & 0,0 & 1,7 & 3,4 & 0,0 & 10,3 & 0,0 & 100 \\
I.2 & 9,0 & 24,4 & 9,0 & 1,3 & 3,8 & 0,0 & 12,8 & 2,6 & 1,3 & 34,6 & 1,3 & 100 \\
I.3 & 0,0 & 38,5 & 7,7 & 0,0 & 0,0 & 7,7 & 15,4 & 7,7 & 0,0 & 23,1 & 0,0 & 100 \\
I.4 & 0,0 & 0,0 & 0,0 & 0,0 & 0,0 & 0,0 & 0,0 & 66,7 & 0,0 & 33,3 & 0,0 & 100 \\
I.5 & 0,0 & 25,0 & 0,0 & 0,0 & 0,0 & 0,0 & 25,0 & 0,0 & 0,0 & 37,5 & 12,5 & 100 \\
I.6 & 14,3 & 21,4 & 7,1 & 0,0 & 0,0 & 0,0 & 21,4 & 7,1 & 0,0 & 28,6 & 0,0 & 100 \\
I.7 & 8,9 & 0,0 & 0,0 & 0,0 & 0,0 & 0,0 & 4,4 & 4,4 & 0,0 & 82,2 & 0,0 & 100 \\
I. 8 & 0,0 & 0,0 & 0,0 & 0,0 & 0,0 & 0,0 & 0,0 & 0,0 & 0,0 & 100 & 0,0 & 100 \\
\hline Total & 14,0 & 25,3 & 5,9 & 0,5 & 1,4 & 0,5 & 9,0 & 4,5 & 0,5 & 37,6 & 0,9 & 100 \\
\hline
\end{tabular}

hacia la práctica deportiva. Se puede afirmar, por tanto, que la continuidad, en su máxima expresión, está asociada con conductas autónomas que no dependen de las amistades.

\section{Discusión y Implicaciones para la Intervención}

La reconstrucción de las experiencias de ocio deportivo de los jóvenes de Bizkaia confirma que existen, en relación a la práctica deportiva, distintos patrones evolutivos que reproducen las diversas direcciones que pueden seguir las trayectorias deportivas juveniles. La identificación y el estudio de estos itinerarios, definidos por las decisiones que los jóvenes toman en cuanto al inicio, continuidad o abandono de su participación deportiva, ha sido clave para detectar los factores psicosociales que modulan la orientación hacia la práctica deportiva regular y el deseo de continuar con la misma. Factores que tal y como se apunta en la literatura especializada han sido identificados como factores determinantes de la práctica deportiva por su capacidad para modular las decisiones en este ámbito, hacia la continuidad o el abandono (Bailey et al., 2005; Fernández, 2007; Nahas et al., 2003; Sallis, Prochaska, \& Taylor, 2000).

Cinco factores emergen como promotores de la práctica deportiva regular (Figura 2); uno, de naturaleza personal, la percepción de barreras, y los cuatro factores 
Tabla 7

Autonomía de las Amistades Como Factor de Continuidad de las PDR

\begin{tabular}{|c|c|c|c|c|c|c|c|c|}
\hline \multirow[b]{2}{*}{$X 2=51,98 ;$ sig $=0,00$} & \multicolumn{2}{|c|}{ Influencia amistades } & \multicolumn{2}{|c|}{ No influencia amistades } & \multicolumn{2}{|c|}{$\mathrm{NS} / \mathrm{NC}$} & \multicolumn{2}{|c|}{ Total } \\
\hline & $\mathrm{N}$ & $\%$ & $\mathrm{~N}$ & $\%$ & $\mathrm{~N}$ & $\%$ & $\mathrm{~N}$ & $\%$ \\
\hline Itinerario 1 & 19 & $32,8 \%$ & 36 & $62,1 \%$ & 3 & $5,2 \%$ & 58 & $100,0 \%$ \\
\hline Itinerario 2 & 61 & $78,2 \%$ & 15 & $19,2 \%$ & 2 & $2,6 \%$ & 78 & $100,0 \%$ \\
\hline Itinerario 3 & 8 & $61,5 \%$ & 3 & $23,1 \%$ & 2 & $15,4 \%$ & 13 & $100,0 \%$ \\
\hline Itinerario 4 & 3 & $100,0 \%$ & 0 & $0,0 \%$ & 0 & $0,0 \%$ & 3 & $100,0 \%$ \\
\hline Itinerario 5 & 4 & $50,0 \%$ & 4 & $50,0 \%$ & 0 & $0,0 \%$ & 8 & $100,0 \%$ \\
\hline Itinerario 6 & 10 & $71,4 \%$ & 4 & $28,6 \%$ & 0 & $0,0 \%$ & 14 & $100,0 \%$ \\
\hline Itinerario 7 & 31 & $68,9 \%$ & 7 & $15,6 \%$ & 7 & $15,6 \%$ & 45 & $100,0 \%$ \\
\hline Itinerario 8 & 2 & $100,0 \%$ & 0 & $0,0 \%$ & 0 & $0,0 \%$ & 2 & $100,0 \%$ \\
\hline Total & 138 & $62,4 \%$ & 69 & $31,2 \%$ & 14 & $6,3 \%$ & 221 & $100,0 \%$ \\
\hline
\end{tabular}

\section{Factores sociales}
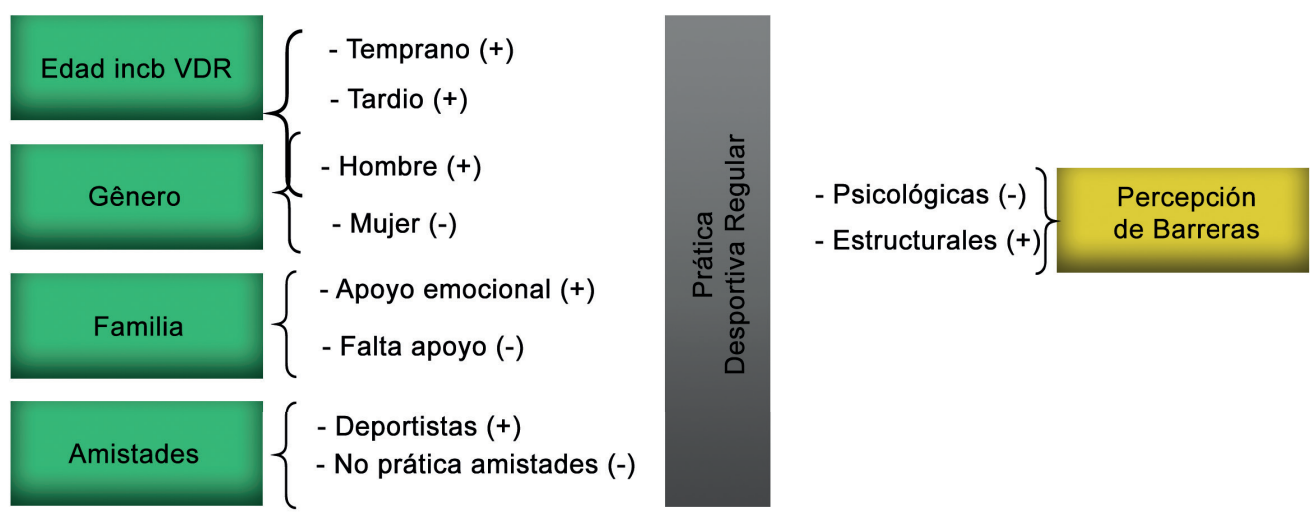

Figura 2. Factores Psicosociales Implicados en la Orientación Hacia la PDR

restantes, de carácter social: la edad, el género, y el apoyo propiciado por agentes sociales significativos del entorno social, fundamentalmente, la familia y las amistades.

La identificación de estos factores implicados en la decisión de tomar parte en la vida deportiva de forma regular proporciona las bases para delinear el perfil tipo de aquellos jóvenes más predispuestos a participar en el ámbito deportivo de esta manera. Este perfil tipo estaría formado preferentemente por:

- hombres,

- iniciados en la VDR en edades tempranas, sobre todo, antes de los 13 años, pero que puede extenderse hasta franjas de edad entre los 17 y 20 años,

- apenas afectados por la percepción de barreras psicológicas,
- arropados por un entorno familiar que valora positivamente el ámbito deportivo y proporciona apoyo emocional a las conductas deportivas de sus hijos,

- insertos en un círculo de amistades, también practicantes deportivos, que les animan a desarrollar este tipo de actividades.

Por su parte, los factores pisocosociales que propician su continuidad son: la concentración en la actividad deportiva desarrollada, la búsqueda de objetivos complejos más allá del entretenimiento, los sentimientos de competencia como principal motivo de continuidad, la inversión personal como consecuencia de largos periodos de VDR y la autonomía de las amistades en la toma de decisiones sobre la persistencia de las propias conductas deportivas. La 
bibliografía especializada proporciona las pautas necesarias para interpretar estos factores implicados en la adherencia deportiva, como indicadores de dos variables principales en torno a las cuales se pueden reagrupar: la motivación intrínseca y el compromiso que de ella se deriva. Ambos aspectos, motivación y compromiso, resultan claves a la hora de saber cómo consolidar la práctica deportiva en los estilos de ocio juveniles. La Figura 3 revela que la importancia de estos cinco factores radica en que afectan directamente a la motivación y al compromiso.

La motivación es, sin duda, uno de los aspectos clave en las conductas de ocio. La Teoría de la Autodeterminación de Deci permite explicar por qué la orientación intrínseca del ocio contribuye a aumentar el sentimiento de autodeterminación en la medida en que las conductas de ocio dan respuesta a necesidades básicas como la competencia, autonomía y relaciones interpersonales inherentes a todo ser humano (Deci, \& Ryan, 1985, 2000a, 2000b). Estos autores destacan la importancia de sustentar las prácticas de ocio en motivos intrínsecos frente a la promoción de conductas de ocio incentivadas por factores externos. Los refuerzos externos, que inicialmente pueden fortalecer y dar continuidad a la participación, a largo plazo generan efectos adversos que acaban minando la motivación intrínseca (Lepper, 1973); mientras que los motivos intrínsecos tales como el sentimiento de competencia, lejos de agotarse tras ser satisfecho, se mantiene e incrementa el atractivo y el interés que la acción de ocio despierta en quien la realiza. De lo anterior, se deduce que es posible intervenir para sustentar el ocio deportivo en motivos internos que favorezcan la continuidad de la práctica. Una afirmación especialmente valiosa para esta investigación, en la que se aboga por favorecer la adherencia deportiva mediante la promoción del valor final del deporte como práctica de ocio, frente a su recurrido valor instrumental (Deci, \& Ryan, 2000a).

Más concretamente, en lo que respecta a la motivación intrínseca hay que tener en cuenta que en esta investigación:

- La percepción de beneficios obtenida mediante la constatación de una mejora en las habilidades deportivas (percepción de competencia), se convierte en los jóvenes continuistas, en uno de los pilares fundamentales de adherencia deportiva. Es, así mismo, muestra de la evolución de los motivos de inicio, desde una motivación de estimulación (centrada en la diversión y en pasarlo bien) a una motivación de logro,

- La relevancia de la percepción de competencia se comprueba al constatar que los jóvenes continuistas persiguen con sus prácticas objetivos elaborados, orientados a la mejora personal a través del logro de retos asociados al aprendizaje y a la consecución de resultados deportivos. Una tendencia que no se detecta en los itinerarios deportivos afectados por el abandono. Se confirma así que ambos objetivos, aprendizaje y resultados deportivos son también fuentes de satisfacción en el ocio deportivo.

- La independencia que los jóvenes continuistas muestran de las conductas deportivas de su círculo de amistades, sugiere la presencia de una fuerte motivación intrínseca, al ser este carácter autónomo, una de las principales expresiones de las conductas autodeterminadas.

El compromiso mantenido con la práctica deportiva emerge en la literatura especializada sobre el tema como uno de los principales predictores de continuidad o abandono (Guillet, Sarrazin, Carpenter, Trouilloud, \& Curry, 2002). Los altos niveles de compromiso se relacionan con

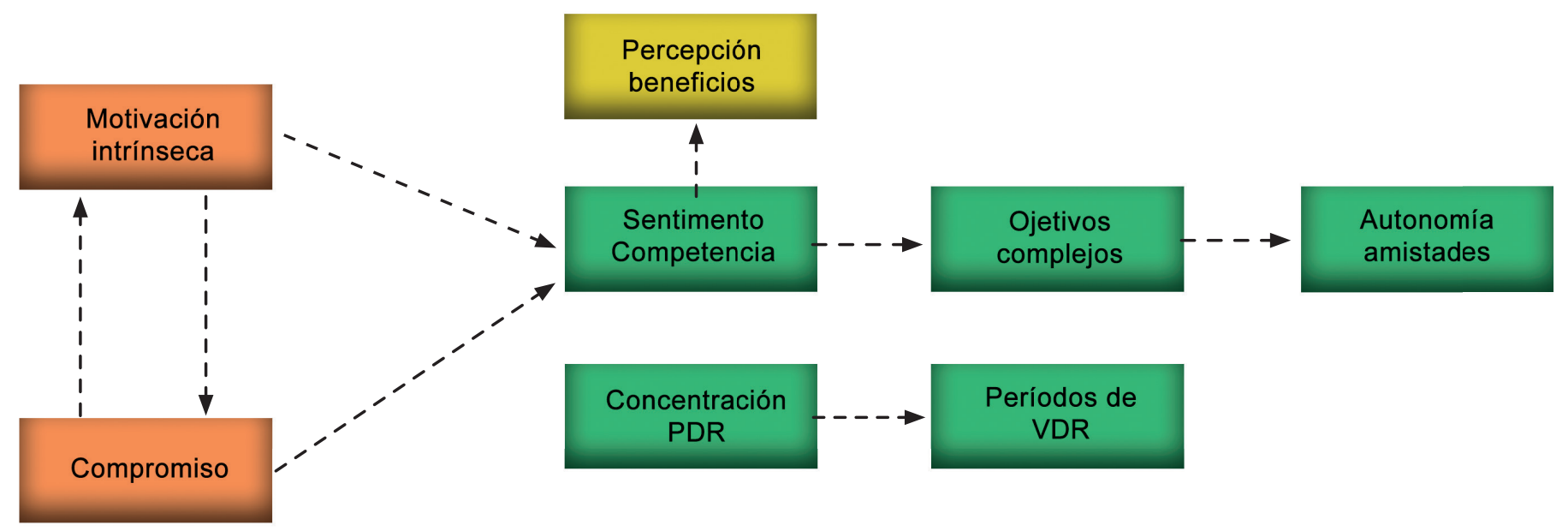

Figura 3. Factores Psicosociales Implicados en la Adherencia Deportiva 
una mayor tendencia a la adherencia, al sustentarse las conductas deportivas en estilos motivacionales intrínsecos. De acuerdo con la Teoría del Intercambio social (Thibaut, \& Kelley, 1959), encontramos que el compromiso deportivo se expresa entre los jóvenes a través de una notable inversión personal, materializada en:

- la concentración en una única actividad deportiva regular (86\%), de la cual se deduce que la actividad deportiva realizada posee un mayor atractivo que otras alternativas de ocio disponibles,

- $\quad$ largos periodos de VDR, cuya media asciende a 6 años y su máxima expresión, a 10 años.

El perfil de los jóvenes continuistas obtenido en este estudio encaja perfectamente con el planteado en otros estudios realizados sobre la relación existente entre compromiso y adherencia (Guillet, Sarrazin, Carpenter, Trouilloud, \& Curry, 2002). Estos jóvenes se perciben a sí mismos como personas más competentes y orientadas a la superación de nuevos retos, más autónomas, menos sensibles al atractivo de otras alternativas de ocio disponibles y con una fuerte carga de inversión personal, en materia deportiva. Se confirma así la pertinencia de teorías como la del Intercambio social (Thibaut, \& Kelley, 1959), según la cual, la continuidad de la práctica deportiva no depende exclusivamente de los sentimientos gratificantes, sino también de otros factores, tales como el compromiso y sus variables antecedentes así como del mantenimiento de una motivación intrínseca, sustentada en la orientación al logro y al crecimiento personal.

Coherentes con la necesidad de integrar el conocimiento obtenido en relación a la totalidad de los factores identificados, en un marco complejo que reconozca la interacción existente entre ellos desde un enfoque multidimensional, se presenta a continuación la Figura 4 en la que se representan los factores implicados en la promoción de la VDR y su continuidad, su naturaleza personal o social, las variables mediante las que estos factores se expresan y las relaciones existentes entre ellos.

En términos generales, cualquier estrategia de intervención orientada a la promoción de la práctica deportiva debería tener en cuenta dos cuestiones básicas fundamentales. En primer lugar, el protagonismo del ocio en la práctica deportiva juvenil. Este aspecto se evidencia como un elemento transversal, siempre prioritario en estas edades y sin embargo, su presencia puede resultar cuestionable en algunas manifestaciones del deporte durante la infancia y la juventud (especialmente, en el deporte escolar). Se deduce de esta afirmación la necesidad de reconocer que la mayoría de las experiencias deportivas durante la infancia y juventud son, ante todo y sobre todo, experiencias de ocio (por ejemplo, el deporte escolar); y una vez reconocido este hecho, actuar en consecuencia, intentado preservar siempre el disfrute que caracteriza las experiencias de ocio. Ahora bien, esto no significa renunciar al logro de resultados o a la mejora de habilidades deportivas puesto que este estudio ha confirmado que ambos aspectos, consecución de resultados deportivos y aprendizaje son fuentes de satisfacción que convenientemente dosificadas y ajustadas a la realidad del protagonista aumentan su interés por la acción. En segundo

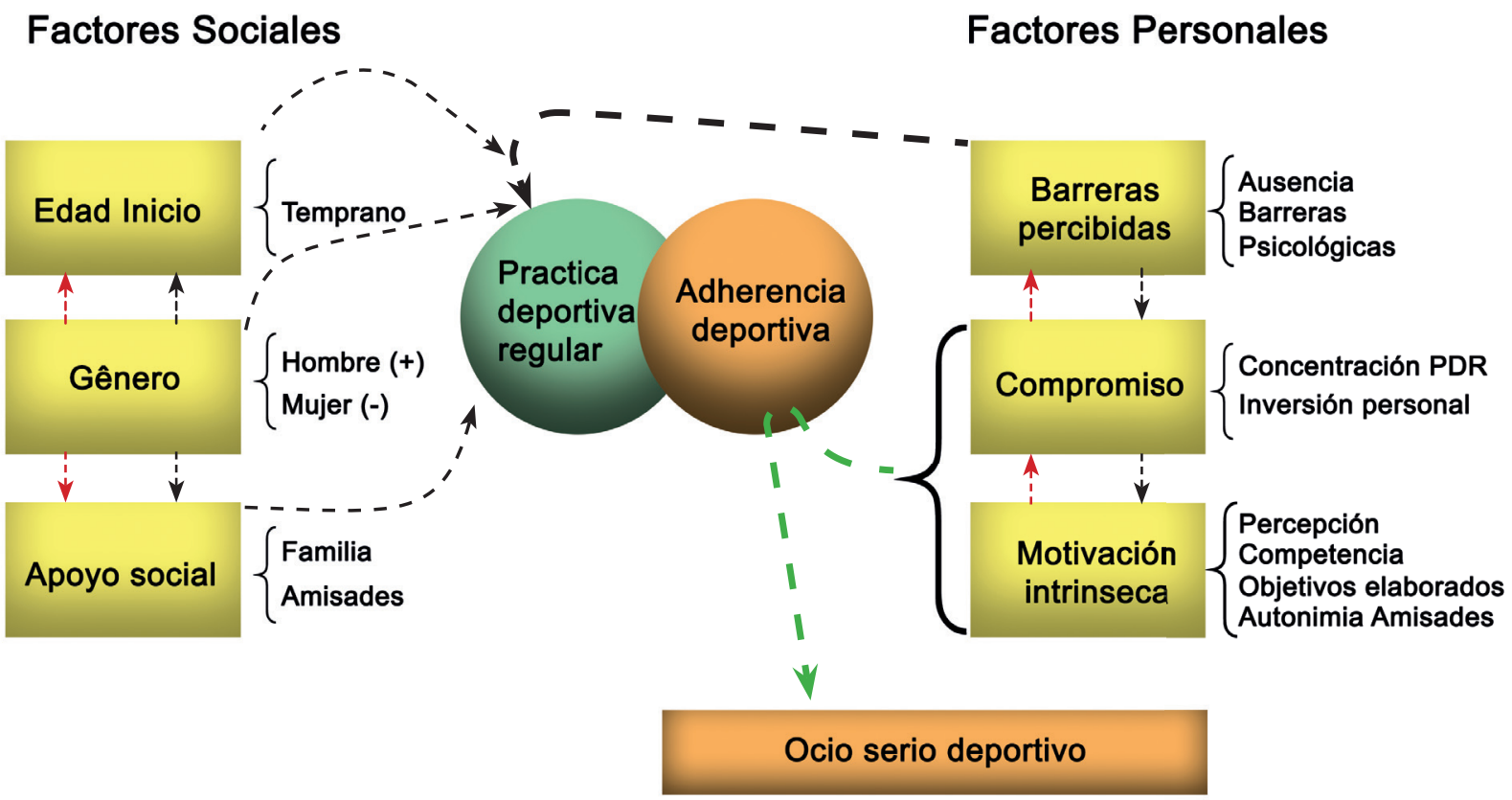

Figura 4. Factores Psicosociales Implicados en la Adherencia Deportiva 
lugar, la identificación de los factores personales y sociales que favorecen la práctica deportiva y su continuidad ha permitido delinear dos perfiles tipo; por un lado, el de aquellos jóvenes especialmente predispuestos a practicar deporte de manera regular; por otro, el de los jóvenes más proclives a continuar haciendo deporte y por tanto, a la adherencia deportiva. Estos perfiles poseen un valor estratégico notorio y deberían ser muy tenidos en cuenta por las y los gestores, educadores, entrenadores y familias que deseen consolidar la práctica deportiva en los estilos de ocio juveniles. El conocimiento de los aspectos que configuran estos perfiles resulta fundamental porque puede orientar la dirección de futuras intervenciones dirigidas a incentivar la participación deportiva de aquellos colectivos menos predispuestos al ocio deportivo y su continuidad entre los jóvenes con mayor tendencia al abandono.

\section{A Modo de Conclusión}

A lo largo del estudio se ha subrayado la importancia de considerar la práctica deportiva como experiencia de ocio valiosa que puede y debe acompañar a la persona a lo largo de la vida, legitimando así su comprensión como proceso materializado en un todo con sentido, denominado itinerario de ocio. La conversión del potencial del ocio deportivo en factor de desarrollo personal depende de las características de dicho proceso. Los estudios realizados en relación a esta cuestión y (Csizksentmihayli, 1998; Cuenca, Aguilar, \& Ortega, 2010; Kleiber, 1999; Stebbins, 2000b; Tinsley, \& Tinsley, 1986) defienden la importancia de vivir un ocio significativo y valioso en cuanto que la satisfacción que propicia deviene de experiencias positivas, caracterizadas por la creciente complejidad de los retos que plantea, el aprendizaje y la superación personal. La posibilidad de desarrollar a lo largo de la vida, experiencias que se aproximen a esta forma sustancial de vivir la práctica deportiva favorece la continuidad y sienta las bases para poder hacer del ocio deportivo un factor de desarrollo personal.

El concepto de itinerario aplicado al ámbito del ocio deportivo emerge como una herramienta válida para desentrañar los factores promotores de la participación deportiva y la adherencia a este ámbito y delinear, en función de dichos factores, la posible evolución de las futuras conductas de ocio deportivo. El conocimiento de tales factores y su influjo sobre las decisiones en materia deportiva permite contemplar la intervención de los agentes implicados como una posibilidad real dirigida a promover la práctica deportiva y su continuidad en los estilos de vida contemporáneos. Pero, sin duda, la aportación más relevante de este trabajo ha sido contribuir al avance de una línea de investigación aún inexplorada, que pretende comprender en pretérito las historias deportivas para poder intervenir y orientar el gusto y el hábito deportivo de diferentes colectivos y el de próximas generaciones hacia formas óptimas de desarrollo personal.

\section{Referências}

Armstrong, G. K., \& Morgan, K. (1998). Stability and change in levels of habitual physical activity in later life. Age and Ageing, 27, 17-23.

Atchley, R. C. (1989). A continuity theory of normal aging. The Gerontologist, 29, 183-190.

Bailey, R., Wellard, I., \& Dismore, H. (2005). Girls participation in physical activities and sport: benefits, patterns, influences and ways forward. Education and Health, 23(1), 3-5. UK:World Health Organization (WHO).

Balaguer, I., Castillo, I., \& Pastor, Y. (2002). Los estilos de vida relacionados con la salud en la adolescencia temprana. In I. Balaguer (Ed.), Estilos de vida en la adolescencia. Valencia: Promolibro.

Beets, M.W., Pitteti, K.H., \& Forlaw, L. (2007). The role of self-efficacy and referent specific social support in promoting rural adolescent girls' physical activity. American Journal of Health Behavior, 31 (3) 227-237.

Carroll, B., \& Alexandris, K. (1997). Perceptions of constraints and strength of motivation: their relation to recreational sport participation. Journal of Leisure Research, 29, 279-299.

Cavill, N., Biddle, S., \& Sallis, J. F. (2001). Health enhaincing physical activuty for young people: Statement for United Kingdom expert consensus conference. Pediatric Exercise Sciences, 13, 12-15.

Cecchini, J.A., Méndez, A., \& Contreras, O.R. (2005). Motivos de abandono de la práctica del deporte juvenil. Cuenca: Universidad de Castilla-La Mancha.

Crawford, D., \& Godbey, G. (1987). Reconceptualizing barriers to family leisure. Leisure Sciences, 9, 119-127.

Csikszentmihalyi, M. (1997). Fluir. Una psicología de la felicidad. Barcelona: Kairós.

Csikszentmihalyi, M. (2000). Ocio y creatividad en el desarrollo humano. In M. Csikszentmihalyi, M. Cuenca, C. Buarque, \& V. Trigo et al., Ocio y Desarrollo. Potencialidades del ocio para el desarrollo humano (pp. 17-32). Documentos de Estudios de Ocio, 16. Bilbao: Universidad de Deusto.

Cuenca Amigo, J. (2013). El valor de la experiencia de ocio 
en la Modernidad Tardía. Documentos de Estudios de Ocio, núm. 48. Bilbao: Universidad de Deusto.

Cuenca, M. (Coord.) (2006). Aproximación multidisciplinar a los Estudios de Ocio. Documentos de Estudios de Ocio, 31. Bilbao: Universidad de Deusto.

Cuenca, M., Aguilar, E., \& Ortega, C. (2010). Ocio para innovar. Documentos de Estudios de Ocio, 42. Bilbao: Universidad de Deusto.

Deci, E.L., \& Ryan, R.M. (1985). Intrinsic motivation and self-determination in human behavior. New York: Plenum.

Deci, E.L., \& Ryan, R.M. (2000a). The support of autonomy and the control of behavior. In E.T. Higgins, \& A.W. Kruglanski, Motivational Science: social and personality perspectives (pp. 128-145). Ann Arbor: Taylor \& Francis.

Deci, E.L., \& Ryan, R.M. (2000b). The "what" and "why" of goal pursuits: Human needs and self-determination of behaviour. Psychological Inquiry, 11, 227-268.

Deloitte S.L. (2009). Encuesta de hábitos deportivos en la Comunidad Autónoma del País Vasco (CAPV). Bilbao: Gobierno Vasco. Departamento de Cultura. Dirección de Deportes.

Duncan, S.C., Duncan, T.E., \& Strycker, L.A. (2005). Sources and types of social support in youth physical activity. Health Psychology, 24(1), 3-10.

Fernández, E. (2007). Barreras para la práctica deportiva de las mujeres españolas durante la infancia y la juventud. ADOZ Revista de Estudios de Ocio, 31, 49-57.

Fraguela, R., Varela, L., Caride, J.A., \& Lera, A. (2009). Deporte y Ocio. Nuevas perspectivas para la acción socioeducativa. Documentos de Estudios de Ocio, 37. Bilbao: Universidad de Deusto.

Frey, B. (2012). Desarrollo humano en Chile 2012. Bienestar subjetivo: el desafio de repensar el desarrollo. Santiago de Chile: Programa de las Naciones Unidas para el Desarrollo.

Ferrando, M. G. (2006). Postmoderrnidad y deporte: entre la individualización y la masificación: encuesta sobre hábitos de los españoles 2005, Madrid: Ministerio de Educación, Cultura y Deporte. Consejo Superior de Deportes.

Ferrando, M. G., \& Llopis, R. (2011). Ideal democrático y bienestar personal. Encuesta sobre los hábitos deportivos en España 2010. Madrid: Consejo Superior de Deportes \& Centro de Investigaciones sociológicas.
Gobierno Vasco, Departamento de Cultura, Dirección General de Deportes (Ed.). (2009). Encuesta de hábitos deportivos en la CAPV. Vitoria-Gasteiz: Publicaciones Gobierno Vasco.

Guillet, E., Sarrazin, P., Carperten, P.J., Trouilloud, D., \& Cury, F. (2002). Predicting persistence or withdrawal in female handballers with social exchange theory. International Journal of Psychology, 37 (2), 93-104.

Hernández, J.L., Velázquez, R., Moya, J.M., Alonso, D., \& Castejón, F.J. (2006). Hábitos de práctica físico-deportiva de los niños, niñas y adolescentes, de su entorno familiar y de su círculo de amistades: estudio de la población española. In J.L. García, M. J. Martínez, \& V. Arufe (Eds.), I Congreso Internacional de Ciencias del Deporte. Pontevedra: Universidad de Vigo. CD-Rom.

Instituto de la Mujer (Ed.). (2006). Actitudes y prácticas deportivas de las mujeres en España (1990-2005). Madrid: Instituto de la Juventud.

Iso-Ahola, S. (1980). The social psychology of leisure. Dubuque: William Brown.

Iso-Ahola, S., Jackson, E., \& Dunn, E. (1994). Starting, ceasing and replacing leisure activities over the lifespan. Journal of Leisure Research, 26, 227-249.

Iwasaki,Y., \& Smale, B. J. A. (1998). Longitudinal analyses of the relationships among life transitions, chronic health problems, leisure, and psychological well-being. Leisure Sciences, 20, 25-52.

Jackson, E.L., \& Dunn, E. (1988). Integrating ceasing participation with other aspects of leisure behavior. Journal of Leisure Research, 20, 31-45.

Kleiber, D. A. (1999). Leisure experience and human development. A dialectical interpretation. New York: Basic Books.

Kleiber, D.A., Walker, G.J., \& Mannell, R.C. (2011). A social psychology of leisure ( $2 \mathrm{a}$ ed.). State College: Venture Publishing.

Lazcano, I., Madariaga, A., \& Doistua, J. (2010). El envejecimiento activo y su incidencia en la experiencia de ocio. ADOZ Revista de Estudios de Ocio, 33, 117147.

Lee, R. E., \& King, A. C. (2003). Discretionary time among older adults: How do physical activity promotion interventions affect sedentary and active behaviors? Annual in Behavioral Medicine, 25, 112-119.

Lepper, M.R., Greene, D., \& Nisbett, R. E. (1973). Undermining children intrinsic interest with extrinsic 
rewards. A test of overjustification hypotesis. Journal of Personality and Social Psychology, 28, 129-137.

Lewis, C.S. (2000). La experiencia de leer. Barcelona: Alba.

Martínez de Quel Pérez, O., Fernández, E., \& Camacho, M.J. (2010, $1^{\circ}$ trimestre). Percepción de dificultades para la práctica de actividad física en chicas adolescentes y su evolución con la edad. Apunts, 99, 92-99.

Martínez Ros, M.T. (1999). Obesidad y actividad fisica. Prevalencia y factores asociados en una muestra representativa de la población de la región de Murcia. Murcia: Universidad de Murcia.

Masnou, M., \& Puig, N. (1995). El acceso al deporte: los itinerarios deportivos. In D. Blázquez (Ed.), $L a$ iniciación deportiva y el deporte escolar (pp. 371-394). Barcelona: Inde Publicaciones.

McGuire, M, Dottavio, F., \& O'Leary, J. (1987). The relationship of early life experiences to later life leisure envolvement. Leisure Sciences, 9, 251-257.

Monteagudo, M.J. (2008). Reconstruyendo la experiencia de ocio: características, condiciones de posibilidad y amenazas en la sociedad de consumo. In. Monteagudo, M.J (Ed.) La experiencia de ocio: una mirada científica desde los Estudios de Ocio (pp. 81-110). Documentos de Estudios de Ocio, 35. Bilbao: Universidad de Deusto.

Monteagudo, M.J. (2011). Los itinerarios de ocio deportivo. Estudio de los jóvenes de Bizkaia (1995-2005). Bilbao: Universidad de Deusto. Tesis Doctoral.

Nahas, M.V., Goldfine, B., \& Collins, M.A. (2003). Determinants of physical activity in adolescents and young adults: the basis for high school and college physical education to promote active lifestyles. Physical Educator, 60(1), 42-56.

Nimrod, G., \& Kleiber, D.A. (2007). Reconsidering change and continuity in later life: toward an innovation theory of successful aging. International Journal of Aging and Human Development, 65, 1-22.

Nussbaum, M.C. (2012). Crear capacidades. Propuesta para el desarrollo humano. Barcelona: Paidós.

Palau, P., Ponseti, X., Gili, M., Borrás, P.A., \& Vidal, J. (2005). Perfil de hábitos deportivos de los preadolescentes de la isala de Mallorca. Revista de Psicología del Deporte, 14(2), 225-236.

Pieron, M., \& Ruiz Juan, F. (2010). Actividad fisicodeportiva y salud. Análisis de los determinantes de la práctica en el alumnado de Enseñanza Secundaria.
Madrid: Ministério de Educación. Consejo superior de Deportes.

Ponseti, X., Vidal, J., Palau, P., \& Borrás, P. A. (2005). Motivos para el inicio, mantenimiento y abandono de la práctica deportiva de los niños y niñas de entre 10 y 14 años de la isla de Mallorca. In M.L. Zagalaz, E.J. Martínez, \& P.A. Latorre (Eds.). I Congreso Internacional y XXII Nacional de Educación Física. Jaén. Universidad de Jaén CD-Rom.

Puig, N., \& Masnou, M. M. (1988). Los itinerarios deportivos de la población juvenil. Revista de Estudios de Juventud, 32, 45-56.

Ramos, R., Sanz, E., Valdemoros, M.A., \& Ponce de León, A. (2010). Sinergias de la educación no formal e informal para consolidar una práctica físico-deportiva en el espacio de ocio. ADOZ. Revista de Estudios de Ocio, 33, 99-116.

Ruiz, J.A.L. (2006). Ocio y tiempo libre. In P. González Blasco (Dir.) \& Fundación Santa María. Informe Jóvenes españoles 2005 (pp. 345-402). Madrid: SM Editorial.

Sallis, J.F., Prochaska, J.J., \& Taylor, W.C. (2000). A review of correlates of physical activity of children and adolescents. Medicine and Science in Sport and Exercise, 32, 963-975.

San Salvador del Valle, R. (2008). O fenómeno do Ócio nas Cidades do século XXI. In M. Cuenca, \& J. Clerton (Orgs.), Ócio para viver no Século XXI (pp.107-124). Fortaleza: As Musas.

Sanz, E. (2005). La práctica físico-deportiva en el tiempo libre en universitarios. Análisis y propuestas de mejora. Logroño: Universidad de La Rioja.

Searle, M.A., Mactavish, J.B., \& Brayley, R.E. (1993). Integrating ceasing participation with other aspects of leisure behavior. Journal of Leisure Research, 25 (4), 389-404.

Stebbins, R.A. (2000a). Un estilo de vida óptimo de ocio: combinar ocio serio y casual en la búsqueda del bienestar personal. In M. Cuenca (Ed.), Ocio humano $y$ desarrollo. Propuestas para el $6^{\circ}$ congreso Mundial de Ocio (pp. 109-116). Bilbao: Universidad de Deusto.

Stebbins, R. A. (2000b). Obligations as an aspect of the leisure experience. Journal of Leisure Research, 32, 152-155.

Stebbins, R. A. (2005). Project-based leisure: theoretical neglected of a common use of free time. Leisure Studies, 24 (1), 1-11. 
Thibaut, J.W., \& Kelley, H.H. (1959). The social psychology of groups. New York: Wiley.

Tinsley, H.E.A., \& Tinsley, D.J. (1986). A theory of atributes, benefits and causes of leisure experience. Leisure Sciences, 8 (1), 1-45.

UK Sport, Sport England and CONI (1999). Sport Participation in Europe: COMPASS 1999. London: UK Sport.

\section{Endereço para correspondência:}

María Jesús Monteagudo Sánchez

Universidad de Deusto. Facultad de Ciencias Sociales y Humanas. Avda. de las Universidades, 27. 48013 Bilbao

E-mail: mjmonte@deusto.es 